Homology, Homotopy and Applications, vol.16(2), 2014, pp.311-344

\title{
DISTANCE FUNCTIONS, CRITICAL POINTS, AND THE TOPOLOGY OF RANDOM ČECH COMPLEXES
}

\author{
OMER BOBROWSKI AND ROBERT J. ADLER
}

(communicated by Gunnar Carlsson)

\begin{abstract}
For a finite set of points $\mathcal{P}$ in $\mathbb{R}^{d}$, the function $d_{\mathcal{P}}: \mathbb{R}^{d} \rightarrow \mathbb{R}^{+}$ measures Euclidean distance to the set $\mathcal{P}$. We study the number of critical points of $d_{\mathcal{P}}$ when $\mathcal{P}$ is a Poisson process. In particular, we study the limit behavior of $N_{k}$ - the number of critical points of $d_{\mathcal{P}}$ with Morse index $k$-as the density of points grows. We present explicit computations for the normalized limiting expectations and variances of the $N_{k}$, as well as distributional limit theorems. We link these results to recent results in $[\mathbf{1 6}, \mathbf{1 7}]$ in which the Betti numbers of the random Cech complex based on $\mathcal{P}$ were studied.
\end{abstract}

\section{Introduction}

For a finite set $\mathcal{P}$ of points in $\mathbb{R}^{d}$ of size $|\mathcal{P}|$, let $d_{\mathcal{P}}: \mathbb{R}^{d} \rightarrow \mathbb{R}^{+}$be the distance function for $\mathcal{P}$, so that

$$
d_{\mathcal{P}}(x):=\min _{p \in \mathcal{P}}\|x-p\|_{2}, \quad x \in \mathbb{R}^{d},
$$

where $\|\cdot\|_{2}$ denotes the Euclidean distance.

The main results of this paper provide considerable information about the asymptotic (in $|\mathcal{P}|$ ) behavior of the critical points (defined below) of $d_{\mathcal{P}}$ when $\mathcal{P}$ is random. While the critical points are, by themselves, intrinsically interesting, knowledge of their behavior also has immediate implications (via Morse theory) for the study of the topology of Čech complexes built over random point sets.

Throughout, we shall concentrate on the situation in which the points in $\mathcal{P}$ are those of a non-homogeneous Possion process with intensity $\lambda_{n}=n f$, where $f$ is a probability density on $\mathbb{R}^{d}$. The mean number of points is therefore $\mathbb{E}\{|\mathcal{P}|\}=n$. Virtually identical results hold when $\mathcal{P}$ is made up of $n$ independent samples from $f$, and proofs in this situation can be found in the PhD thesis [6].

O.B. was supported in part by the Adams Fellowship Program of the Israel Academy of Sciences and Humanities and FP7-ICT-318493-STREP, TOPOSYS. R.A. was supported in part by AFOSR FA8655-11-1-3039 and ERC 2012 Advanced Grant 20120216, URSAT.

Received September 9, 2013, revised June 29, 2014; published on November 24, 2014.

2010 Mathematics Subject Classification: 60D05, 60F05, 60G55, 55U10, 58K05.

Key words and phrases: distance function, critical points, Morse index, Čech complex, Poisson process, central limit theorem, Betti numbers.

Article available at http://dx.doi.org/10.4310/HHA.2014.v16.n2.a18

Copyright (C) 2014, International Press. Permission to copy for private use granted. 
Most of what we shall have to say will concentrate on the distance function in neighborhoods of radius $r_{n}$ around $\mathcal{P}$, when $n \rightarrow \infty$ and $r_{n} \rightarrow 0$. Our main results give expressions for the normalized asymptotic means and variances of $N_{k, n}$-the number of critical points with index $k$ appearing within distance $r_{n}$ from $\mathcal{P}$-along with various distributional limit results. The limit distributions are of different kinds, and, depending on delicate relationships between $d, k, r_{n}$, and $n$, provide limits that may be Gaussian, Poisson, or deterministic, while also exhibiting a range of critical phenomena. Note that there are various notions of convergence used in probability theory, and so in Appendix A we provide definitions of the notions that we need. Our main results on critical points are described in detail in Section 3. However, before stating the results, we first need to describe precisely how to define the critical points, along with their indices, for the distance function. The difficulty lies in the fact that the distance function is not everywhere differentiable. We shall do this in the following section.

In Section 4 we shall discuss the relationship between $N_{k, n}$ and the Betti numbers of a special simplicial complex, the Čech complex, based on $\mathcal{P}$. The homology of the Cech complex is closely related to the neighborhood set, or $r_{n}$-tube around $\mathcal{P}$,

$$
\mathcal{B}_{n}:=\bigcup_{p \in \mathcal{P}} B_{r_{n}}(p)
$$

where $B_{r_{n}}(p)$ is the $d$-ball of radius $r_{n}$ around $p$. What we shall see in Section 4 is that, if $r_{n}$ is too small, then the individual balls in (1.2) will generally fail to intersect, and the topology will be approximately that of a large number of disjoint points. This is occasionally referred to as the "dust" or "sparse" regime, although there does not yet seem to be a universally accepted term. If $r_{n}$ decays too slowly, then the balls will connect and the topology of $\mathcal{B}_{n}$ will be that of a single ball. At the (phase) transition $\mathcal{B}_{n}$ will have a percolative-like structure, and so we call this the percolation phase, which is also known as the "thermodynamic limit." Each of these phases exhibits different limit behavior, with even more subtle differences possible within phases depending on interactions between parameters.

Translating our results about critical points into statements about the (algebraic) topological structure of $\mathcal{B}_{n}$, as $n \rightarrow \infty$, will also allow us to compare them to other results currently in the literature (primarily $[\mathbf{1 6}, \mathbf{1 7}]$ ). The one comment that we already make at this stage, however, is that we can provide a much richer set of results for the asymptotic behavior of numbers of critical points than is currently available for the Betti numbers of these Čech complexes. Indeed, we can also provide some topological results via critical points that are not yet available with a direct topological approach. For example, we are able to compute properties of the Euler characteristic $\chi_{n}$ of the complex, and can show (see Corollary 4.2 for details) that there exist functions $\gamma_{k}$ such that

$$
\lim _{n \rightarrow \infty} n^{-1} \mathbb{E}\left\{\chi_{n}\right\}= \begin{cases}1 & n r_{n}^{d} \rightarrow 0 \\ 1+\sum_{k=1}^{d}(-1)^{k} \gamma_{k}(\lambda) & n r_{n}^{d} \rightarrow \lambda \in(0, \infty), \\ 0 & n r_{n}^{d} \rightarrow \infty\end{cases}
$$

Moreover, when $n r_{n}^{d} \rightarrow \infty$ and $n r_{n}^{d} \geqslant D^{\star} \log n$ for some $D^{\star}$ (cf. Proposition 3.9), then $\mathbb{E}\left\{\chi_{n}\right\} \rightarrow 1$. 
The remainder of the paper contains the proofs of the results in Sections 3 and 4. These are organized in a number of sections and appendices so as to make them as user friendly as possible. Many of the proofs rely on techniques in the theory of random geometric graphs as developed in $[\mathbf{2 3}]$.

Finally, a few words on motivation. There is considerable current interest in the study, from a topological, homological point of view of random structures such as graphs and simplicial complexes. Some recent references are $[\mathbf{3}, \mathbf{5 ,} 7, \mathbf{1 0}, \mathbf{1 8}, \mathbf{2 4}]$ with two reviews, from different aspects, in [1] and [12]. Many of these papers find their raison d'être in essentially statistical problems, in which data generates these structures. An important example appears in the papers [22, 21], which show that the homology of an unknown manifold can be recovered, with high probability, by looking at the homology of the union of balls around the points of random samples (or equivalently, at the homology of the Čech complex generated by the sampling points on the manifold) with or without additional noise. The homological theme of these papers, which considers manifolds as being "close" if their homologies are the same, seems particularly promising for situations in which the manifold of interest is embedded in a space of much higher dimension than itself; i.e., in dimension reduction problems and in manifold learning.

The approach adopted in this paper shares the motivation of the others listed above, but as already noted, by adopting a Morse theoretic point of view based on critical points of the distance function, obtains a more internally complete theory. Further, as mentioned above and shown later, it often gives some information on global topological invariants, such as Betti numbers. However, being based on critical points, this approach is naturally limited in its ability to reveal the full picture about the global topological invariants of random complexes.

\section{Acknowledgments}

We thank Shmuel Weinberger for introducing us to this problem, and also thank Yuliy Baryshnikov, Matthew Strom Borman, Matthew Kahle, and Shmuel Weinberger, for many useful discussions in the earlier stages of our work.

\section{Critical points of the distance function}

Critical points of smooth functions have been studied since the earliest days of calculus, but took on significant additional importance following the development of Morse theory (e.g., $[\mathbf{1 9}, \mathbf{2 0}]$ ), which tied them closely to the homologies of manifolds, a topic that we shall discuss briefly in Section 4. At this point we note that if $\mathcal{M}$ is a nice (closed, differentiable) $n$-dimensional manifold, and $f: \mathcal{M} \rightarrow \mathbb{R}$ a nice (Morse) function, then a point $c$ is called a critical point if $\nabla f(c)=0$. A non-degenerate critical point is one for which the Hessian matrix $H_{f}(c)$ is non-singular. The Morse index $k \in\{0,1, \ldots, n\}$ of a non-degenerate critical point $c$ is then the number of negative eigenvalues of $H_{f}(c)$. These points, along with their indices, provide one of the main links between differential and algebraic topology.

Classical Morse theory does not directly apply to the distance function mainly because it is not everywhere differentiable. However, when the set $\mathcal{P}$ is finite, one can still define a notion of non-degenerate critical points for the distance function $d_{\mathcal{P}}$, as well as their Morse index. It turns out that, even in this case, knowledge of 
the critical points and their indices allows one to deduce topological properties of the related Čech complexes. We shall see how to do this later in Section 4, but for now we need some definitions. Our arguments follow from the results presented in [11]. While the distance function served as the main motivation in [11], the results presented there are given in the more general context of "min-type" functions. Here, we specialize those results to the case of the distance function.

Given a finite set of points $\mathcal{P} \subset \mathbb{R}^{d}$, and defining the distance function $d_{\mathcal{P}}$ (1.1), we start with the local (and global) minima of $d_{\mathcal{P}}$; viz. the points of $\mathcal{P}\left(\right.$ where $\left.d_{\mathcal{P}}=0\right)$, and call these critical points with index 0. For higher indices, we have the following definition.

Definition 2.1. A point $c \in \mathbb{R}^{d}$ is a critical point of $d_{\mathcal{P}}$ with index $1 \leqslant k \leqslant d$ if there exists a subset $\mathcal{Y}$ of $k+1$ points in $\mathcal{P}$ such that:

1. $\forall y \in \mathcal{Y}: d_{\mathcal{P}}(c)=\|c-y\|_{2}$, and $\forall p \in \mathcal{P} \backslash \mathcal{Y}:\|c-p\|_{2}>d_{\mathcal{P}}(p)$.

2. The points in $\mathcal{Y}$ are in general position (i.e., the $k+1$ points of $\mathcal{Y}$ do not lie in a $(k-1)$-dimensional affine space).

3. $c \in \operatorname{conv}^{\circ}(\mathcal{Y})$, where $\operatorname{conv}^{\circ}(\mathcal{Y})$ is the interior of the convex hull of $\mathcal{Y}$ (an open $k$-simplex in this case).

The first condition implies that $d_{\mathcal{P}} \equiv d_{\mathcal{Y}}$ in a small neighborhood of $c$. The second condition implies that the points in $\mathcal{Y}$ lie on a unique $(k-1)$-dimensional sphere. We shall use the following notation:

$$
\begin{aligned}
& S(\mathcal{Y})=\text { the unique }(k-1) \text {-dimensional sphere containing } \mathcal{Y} \\
& C(\mathcal{Y})=\text { the center of } S(\mathcal{Y}) \text { in } \mathbb{R}^{d} \\
& R(\mathcal{Y})=\text { the radius of } S(\mathcal{Y}), \\
& B(\mathcal{Y})=\text { the open ball in } \mathbb{R}^{d} \text { with radius } R(\mathcal{Y}) \text { centered at } C(\mathcal{Y}) .
\end{aligned}
$$

Note that $S(\mathcal{Y})$ is a $(k-1)$-dimensional sphere, whereas $B(\mathcal{Y})$ is a $d$-dimensional ball. Obviously, $S(\mathcal{Y}) \subset B(\mathcal{Y})$, but unless $k=d, S$ is not the boundary of $B$. Since the critical point $c$ in Definition 2.1 is equidistant from all the points in $\mathcal{Y}$, we have that $c=C(\mathcal{Y})$. Thus, we say that $c$ is the unique index $k$ critical point generated by the $k+1$ points in $\mathcal{Y}$. The last statement can be rephrased as follows:

Lemma 2.2. A subset $\mathcal{Y} \subset \mathcal{P}$ of $k+1$ points in general position generates an index $k$ critical point if, and only if, the following two conditions hold:

$$
\begin{array}{ll}
\text { CP1 } & C(\mathcal{Y}) \in \operatorname{conv}^{\circ}(\mathcal{Y}), \\
\text { CP2 } & \mathcal{P} \cap B(\mathcal{Y})=\emptyset .
\end{array}
$$

Furthermore, the critical point is $C(\mathcal{Y})$ and the critical value is $R(\mathcal{Y})$.

Figure 1 depicts the generation of an index 2 critical point in $\mathbb{R}^{2}$ by subsets of 3 points. We shall also be interested in critical points $c$ that are within distance $\epsilon$ from $\mathcal{P}$, i.e., $d_{\mathcal{P}}(c) \leqslant \epsilon$. This adds a third condition,

CP3 $R(\mathcal{Y}) \leqslant \epsilon$.

The following indicator functions, related to CP1-CP3, will appear often. 

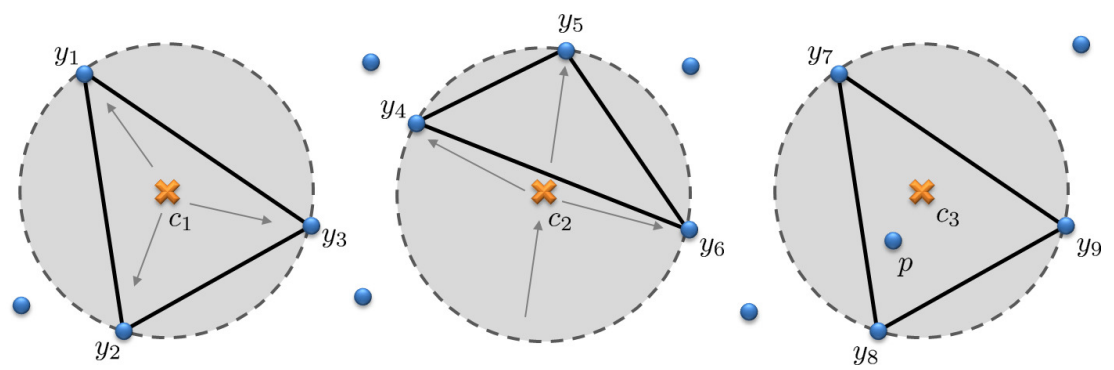

Figure 1: Generating a critical point of index 2 in $\mathbb{R}^{2}$ (i.e., a maximum point). The small disks are the points of $\mathcal{P}$. We examine three subsets of $\mathcal{P}: \mathcal{Y}_{1}=\left\{y_{1}, y_{2}, y_{3}\right\}$, $\mathcal{Y}_{2}=\left\{y_{4}, y_{5}, y_{6}\right\}$, and $\mathcal{Y}_{3}=\left\{y_{7}, y_{8}, y_{9}\right\} . S\left(\mathcal{Y}_{i}\right)$ are the dashed circles, whose centers are $C\left(\mathcal{Y}_{i}\right)=c_{i}$. The shaded balls are $B\left(\mathcal{Y}_{i}\right)$, and the interior of the triangles are $\operatorname{conv}^{\circ}\left(\mathcal{Y}_{i}\right)$. (1) We see that both $C\left(\mathcal{Y}_{1}\right) \in \operatorname{conv}^{\circ}\left(\mathcal{Y}_{1}\right)(\mathbf{C P 1})$ and $\mathcal{P} \cap B\left(\mathcal{Y}_{1}\right)=\emptyset(\mathbf{C P 2})$. Hence $c_{1}$ is a critical point of index 2. (2) $C\left(\mathcal{Y}_{2}\right) \notin \operatorname{conv}^{\circ}\left(\mathcal{Y}_{2}\right)$, which means that $(\mathbf{C P 1})$ does not hold, and therefore $c_{2}$ is not a critical point (as can be observed from the flow arrows). (3) $C\left(\mathcal{Y}_{3}\right) \in \operatorname{conv}^{\circ}\left(\mathcal{Y}_{3}\right)$, so (CP1) holds. However, we have $\mathcal{P} \cap B\left(\mathcal{Y}_{3}\right)=\{p\}$, so (CP2) does not hold, and therefore $c_{3}$ is also not a critical point. Note that in a small neighborhood of $c_{3}$ we have $d_{\mathcal{P}} \equiv d_{\{p\}}$, completely ignoring the existence of $\mathcal{Y}_{3}$.

Definition 2.3. Using the notation above,

$$
\begin{aligned}
h(\mathcal{Y}) & :=\mathbb{1}\left\{C(\mathcal{Y}) \in \operatorname{conv}^{\circ}(\mathcal{Y})\right\} & (\mathbf{C P 1}) \\
h_{\epsilon}(\mathcal{Y}) & :=h(\mathcal{Y}) \mathbb{1}_{[0, \epsilon]}(R(\mathcal{Y})) & (\mathbf{C P 1}+\mathbf{C P 3}) \\
g_{\epsilon}(\mathcal{Y}, \mathcal{P}) & :=h_{\epsilon}(\mathcal{Y}) \mathbb{1}\{\mathcal{P} \cap B(\mathcal{Y})=\emptyset\} & (\mathbf{C P 1}+\mathbf{C P 2}+\mathbf{C P 3})
\end{aligned}
$$

\section{Main results}

Let $f$ be a bounded probability density function on $\mathbb{R}^{d}$, which we assume to be bounded. This assumption will remain in force throughout the paper, without further comment. Let $\mathcal{P}_{n}$ be a spatial Poisson process on $\mathbb{R}^{d}$ with intensity function $\lambda_{n}=n f$. Denote by $\mathcal{C}(n, k)$ the sets of critical points with index $k$ of $d_{\mathcal{P}_{n}}$. Let $\left\{r_{n}\right\}_{n=1}^{\infty}$ be a sequence of positive numbers with $\lim _{n \rightarrow \infty} r_{n}=0$, and define

$$
N_{k, n}:=\#\left\{c \in \mathcal{C}(n, k): d_{\mathcal{P}_{n}}(c) \leqslant r_{n}\right\} .
$$

Our main goal is to study the limits of $N_{k, n}$ as $n \rightarrow \infty$. Since $\mathbb{E}\left\{N_{0, n}\right\} \equiv n$ (the minima are the points of $\mathcal{P}_{n}$ ), we shall only be interested in $1 \leqslant k \leqslant d$. The results split into three main regimes, depending on the rate of convergence of $r_{n}$ to zero, specifically, on the limit of the term $n r_{n}^{d}$.

A word on notation: In the formulae presented below, for $g:\left(\mathbb{R}^{d}\right)^{k+1} \rightarrow \mathbb{R}$ and $\mathbf{y}=\left(y_{1}, \ldots, y_{k}\right) \in\left(\mathbb{R}^{d}\right)^{k}$ we write $g(0, \mathbf{y})$ for $g\left(0, y_{1}, \ldots, y_{k}\right)$. 


\subsection{The subcritical range $\left(n r_{n}^{d} \rightarrow 0\right)$}

This range is also known as the "dust phase," for reasons that will become clearer later, when we discuss Čech complexes. We start with the limiting mean.

Theorem 3.1 (Limit mean). If $n r_{n}^{d} \rightarrow 0$, then for $1 \leqslant k \leqslant d$,

$$
\lim _{n \rightarrow \infty}\left(n^{k+1} r_{n}^{d k}\right)^{-1} \mathbb{E}\left\{N_{k, n}\right\}=\mu_{k}
$$

where

$$
\mu_{k}=\frac{1}{(k+1) !} \int_{\mathbb{R}^{d}} f^{k+1}(x) d x \int_{\left(\mathbb{R}^{d}\right)^{k}} h_{1}(0, \mathbf{y}) d \mathbf{y},
$$

and $h_{1}$ is defined in (2.6).

In general, as is common for results of this nature, it is difficult to express this integral in a more transparent form. However, when $k=1, \mathbf{y}$ contains only a single point, and so $h \equiv 1$ and $R(0, \mathbf{y})=\|\mathbf{y}\| / 2$. Therefore, $h_{1}(0, \mathbf{y})=\mathbb{1}\{\|\mathbf{y}\| \leqslant 2\}$, yielding $\mu_{1}=2^{d-1} \omega_{d} \int_{\mathbb{R}^{d}} f^{2}(x) d x$, where $\omega_{d}$ is the volume of the unit ball in $\mathbb{R}^{d}$. Some numerics for other cases are given below.

The observation that, for a specific choice of $r_{n}$, there is at most one $\alpha \in[1, d]$ such that $\lim _{n \rightarrow \infty} n^{\alpha+1} r_{n}^{d \alpha} \in(0, \infty)$ leads to the important fact that there is a "critical" index, $k_{c}:=\lfloor\alpha\rfloor$, such that

$$
k<k_{c} \Rightarrow \lim _{n \rightarrow \infty} \mathbb{E}\left\{N_{k, n}\right\}=\infty, \quad k>k_{c} \Rightarrow \lim _{n \rightarrow \infty} \mathbb{E}\left\{N_{k, n}\right\}=0,
$$

with any value in $(0, \infty]$ possible at $k=k_{c}$. That is, there is phase transition occurring within the subcritical regime itself. Similar regimes, with identical limits, appear for asymptotic variances.

Theorem 3.2 (Limit variance). If $n r_{n}^{d} \rightarrow 0$, then for $1 \leqslant k \leqslant d$,

$$
\lim _{n \rightarrow \infty}\left(n^{k+1} r_{n}^{d k}\right)^{-1} \operatorname{Var}\left(N_{k, n}\right)=\mu_{k} .
$$

Not surprisingly, the three regimes also yield different limit distributions.

Theorem 3.3 (Limit distribution). Let $n r_{n}^{d} \rightarrow 0$, and $1 \leqslant k \leqslant d$.

1. If $\lim _{n \rightarrow \infty} n^{k+1} r_{n}^{d k}=0$, then

$$
N_{k, n} \stackrel{L^{2}}{\longrightarrow} 0 .
$$

2. If $\lim _{n \rightarrow \infty} n^{k+1} r_{n}^{d k}=\alpha \in(0, \infty)$, then

$$
N_{k, n} \stackrel{\mathcal{L}}{\rightarrow} \text { Poisson }\left(\alpha \mu_{k}\right) .
$$

3. If $\lim _{n \rightarrow \infty} n^{k+1} r_{n}^{d k}=\infty$, then

$$
\frac{N_{k, n}-\mathbb{E}\left\{N_{k, n}\right\}}{\left(n^{k+1} r_{n}^{d k}\right)^{1 / 2}} \stackrel{\mathcal{L}}{\rightarrow} \mathcal{N}\left(0, \mu_{k}\right) .
$$

As above, for a specific choice of $r_{n}$, there is going to be at most a single $k_{c}$ for which the Poisson limit applies. Otherwise $N_{k, n}$ converges either to zero or infinity. Thus, in the subcritical regime, the picture is that $n=N_{0, n} \gg N_{1, n} \gg \cdots \gg N_{k_{c}, n}$, while for $k>k_{c}$ the value of $N_{k, n}$ will be zero with high probability, which increases with $k$. 
3.2. The critical and supercritical ranges $\left(n r_{n}^{d} \rightarrow \lambda \in(0, \infty]\right)$

We now look at the critical $\left(n r_{n}^{d} \rightarrow \lambda \in(0, \infty)\right)$ and supercritical $\left(n r_{n}^{d} \rightarrow \infty\right)$ regimes. While there are differences between the two regimes, the general outline of the results is the same. In both, the correct scaling for $N_{k, n}$ is $n$ (as opposed to $n^{k+1} r_{n}^{d k}$ in the subcritical range). Consequently, the limit results are similar for all the indices.

The supercritical regime is significantly more difficult to analyze than either the critical or subcritical, and we shall require an additional assumption for this case, which necessitates a definition.

Definition 3.4. Let $f: \mathbb{R}^{d} \rightarrow \mathbb{R}$ be a probability density function. We say that $f$ is lower bounded if it has compact support and $f_{\min }:=\inf \{f(x): x \in \operatorname{supp}(f)\}>0$.

Henceforth, when dealing with the supercritical phase, we always assume that $f$ is a lower bounded probability density, and that $\operatorname{supp}(f)$ is convex. It is not clear at this point if these are necessary conditions, or a consequence of our proofs.

Theorem 3.5 (Limit mean). If $n r_{n}^{d} \rightarrow \lambda \in(0, \infty]$, then, for $1 \leqslant k \leqslant d$,

$$
\lim _{n \rightarrow \infty} n^{-1} \mathbb{E}\left\{N_{k, n}\right\}=\gamma_{k}(\lambda)
$$

where

$$
\begin{aligned}
\gamma_{k}(\lambda) & =\frac{\lambda^{k}}{(k+1) !} \int_{\left(\mathbb{R}^{d}\right)^{k+1}} f^{k+1}(x) h_{1}(0, \mathbf{y}) e^{-\lambda \omega_{d} R^{d}(0, \mathbf{y}) f(x)} d \mathbf{y} d x, \\
\gamma_{k}(\infty) & =\lim _{\lambda \rightarrow \infty} \gamma_{k}(\lambda)=\frac{1}{(k+1) !} \int_{\left(\mathbb{R}^{d}\right)^{k}} h(0, \mathbf{y}) e^{-\omega_{d} R^{d}(0, \mathbf{y})} d \mathbf{y},
\end{aligned}
$$

$\omega_{d}$ is the volume of the unit ball in $\mathbb{R}^{d}$, and $R, h$, and $h_{1}$ are defined in (2.3), (2.5), and (2.6), respectively.

Again, these terms can be evaluated for $k=1$, in which case

$$
\begin{aligned}
\gamma_{1}(\lambda) & =\frac{\lambda}{2} \int_{\mathbb{R}^{d}} \int_{\|y\|_{2} \leqslant 2} f^{2}(x) e^{-\lambda \omega_{d} 2^{-d}\|y\|_{2}^{d} f(x)} d y d x, \\
\gamma_{1}(\infty) & =\frac{1}{2} \int_{\mathbb{R}^{d}} e^{-\omega_{d} 2^{-d}\|y\|_{2}^{d}} d y=2^{d-1} .
\end{aligned}
$$

For a uniform distribution on a compact set $D \subset \mathbb{R}^{d}$ it is easy to show that $\gamma_{1}(\lambda)$ is given by

$$
\gamma_{1}(\lambda)=2^{d-1}\left(1-e^{-\lambda \omega_{d} / \operatorname{Vol}(D)}\right),
$$

from which it is easy to check that $\gamma_{1}(\lambda) \rightarrow \gamma_{1}(\infty)$ as $\lambda \rightarrow \infty$. For higher indices, we have no analytic way to compute $\gamma_{k}(\lambda)$. However, it can be evaluated numerically, and an example is given in Figure 2 for the uniform distribution on $[0,1]^{3}$. Note that, in that example, $\gamma_{0}(\infty)-\gamma_{1}(\infty)+\gamma_{2}(\infty)-\gamma_{3}(\infty) \approx 0$. This is not a coincidence, and the explanation for this phenomenon will be given in Section 4.3, where we discuss the mean Euler characteristic of Cech complexes.

Recall that, in the subcritical phase, the limit mean and the limit variance were exactly the same. For other phases, this is no longer true. 


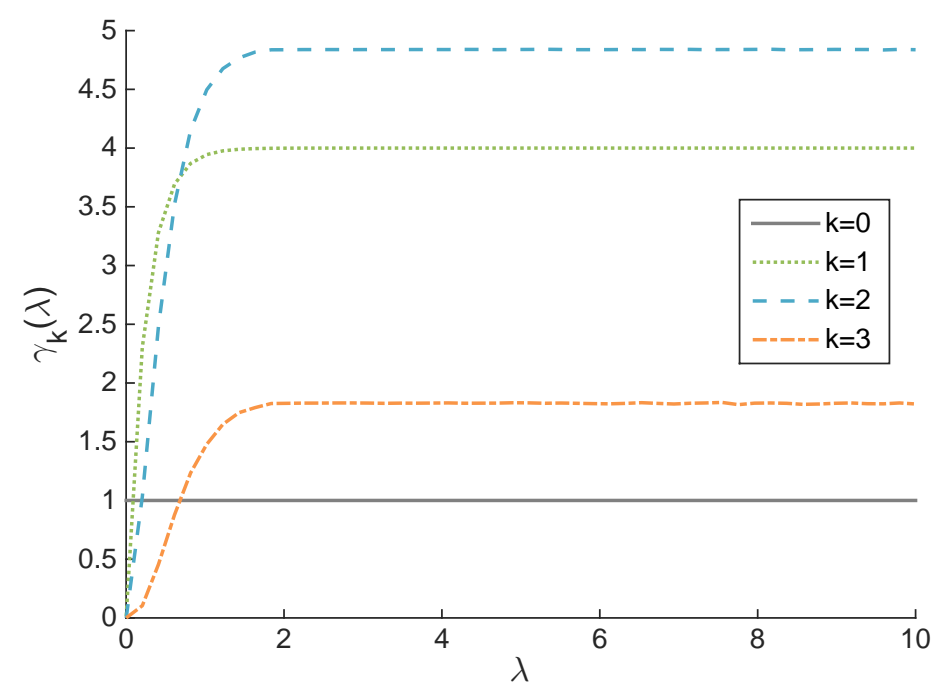

Figure 2: The $\gamma_{k}(\lambda)$ function. In this example $d=3$, and $f(x)$ is the uniform density on $[0,1]^{3}$. For $k=0$ we know that $n^{-1} N_{0, n}=1$, and for $k=1$ we have an explicit formula in (3.5). For $k=2,3$ we used numerical integration followed by some smoothing.

Theorem 3.6 (Limit variance). If $n r_{n}^{d} \rightarrow \lambda \in(0, \infty]$ and $1 \leqslant k \leqslant d$,

$$
\lim _{n \rightarrow \infty} n^{-1} \operatorname{Var}\left(N_{k, n}\right)=\sigma_{k}^{2}(\lambda)>0
$$

The expression defining $\sigma_{k}^{2}(\lambda)$ is rather complicated, and can be found in (8.4). Note that as an immediate corollary of Theorems 3.5 and 3.6, we have the "law of large numbers" that $n^{-1} N_{k, n} \stackrel{L^{2}}{\longrightarrow} \gamma_{k}(\lambda)$. In addition, we also prove the following "central limit theorem" (CLT).

Theorem 3.7 (CLT). If $n r_{n}^{d} \rightarrow \lambda \in(0, \infty]$, then for $1 \leqslant k \leqslant d$,

$$
\frac{N_{k, n}-\mathbb{E} N_{k, n}}{\sqrt{n}} \stackrel{\mathcal{L}}{\rightarrow} \mathcal{N}\left(0, \sigma_{k}^{2}(\lambda)\right) .
$$

To conclude this section, we note an interesting result which is unique to the supercritical regime, for which we define $N_{k, n}^{(g)}:=|\mathcal{C}(n, k)|$, the "global" number of critical points of the distance function $d_{\mathcal{X}_{n}}$ in $\mathbb{R}^{d}$ (i.e., without requiring $($ CP3)). We note first that $N_{k, n}$ and $N_{k, n}^{(g)}$ have identical asymptotic behaviors, at least at the level of their first two moments and CLT:

Theorem 3.8. Let $f$ be lower bounded with a convex support. Then, for $1 \leqslant k \leqslant d$,

$$
\lim _{n \rightarrow \infty} n^{-1} \mathbb{E}\left\{N_{k, n}^{(g)}\right\}=\gamma_{k}(\infty), \quad \lim _{n \rightarrow \infty} n^{-1} \operatorname{Var}\left(N_{k, n}^{(g)}\right)=\sigma_{k}^{2}(\infty),
$$


and

$$
\frac{N_{k, n}^{(g)}-\mathbb{E}\left\{N_{k, n}^{(g)}\right\}}{\sqrt{n}} \stackrel{\mathcal{L}}{\rightarrow} \mathcal{N}\left(0, \sigma_{k}^{2}(\infty)\right) .
$$

An obvious corollary of Theorem 3.8 is that $n^{-1} \mathbb{E}\left\{N_{k, n}^{(g)}-N_{k, n}\right\} \rightarrow 0$. However, much more is true:

Proposition 3.9. Under the conditions of Theorem 3.8, and if $n r_{n}^{d} \geqslant D^{\star} \log n$, for sufficiently large ( $f$-dependent) $D^{\star}$, then, for $1 \leqslant k \leqslant d$,

$$
\lim _{n \rightarrow \infty} \mathbb{E}\left\{\left|N_{k, n}^{(g)}-N_{k, n}\right|\right\}=0 .
$$

Thus, in the supercritical phase, the slow decrease of the radii $r_{n}$ implies that the global and the local number of critical points are ultimately equal with high probability, despite the fact that both grow to infinity with increasing $n$. This is an interesting result, and will turn out to be important when we discuss the Euler characteristic of the Cech complex in the next section. The equality between the local and global counts can be explained if we study how well $\operatorname{supp}(f)$ is covered by the random balls of radius $r_{n}$. Denoting by $C_{n}$ the event that $\operatorname{supp}(f) \subset \bigcup_{X \in \mathcal{P}} B_{r_{n}}(X)$, similar methods as in $[\mathbf{1 3}, \mathbf{1 5}, \mathbf{2}]$ can then be applied to show that if $n r_{n}^{d} \geqslant D \log n$, then $\mathbb{P}\left(C_{n}\right) \rightarrow 1$. Thus, under the assumptions of Proposition 3.9, the support of $f$ is completely covered by the $r_{n}$-balls. Since all the critical points lie within the support, we have that they all should be accounted for in $N_{k, n}$. Note that (3.7) relies heavily on the assumed convexity of $\operatorname{supp}(f)$. For example, take $f$ to be the uniform density on the annulus $A=\left\{x \in \mathbb{R}^{2}: 1 \leqslant|x| \leqslant 2\right\}$. Then, for $n$ large enough, we would expect to have a maximum point (index 2) close to the origin. This critical point will be accounted for in $N_{2, n}^{(g)}$, but will be ignored by $N_{2, n}$, since its distance to $\mathcal{X}_{n}$ is greater than 1 . Thus, we would expect that $\mathbb{E}\left\{\left|N_{2, n}^{(g)}-N_{2, n}\right|\right\} \rightarrow 1$, which contradicts (3.7).

\section{Random Čech complexes}

As mentioned already a number of times, the results of the previous section regarding critical points of the distance function have implications for the homology and Betti numbers of certain random Čech complexes, and so are related to recent results of [16] and [17]. Our plan in this section is to describe these complexes and then the connections. We shall assume that the reader either has a basic grounding in algebraic topology at the level of the first two chapters of [14] or is prepared to accept a definition of the $k$-th Betti number $\beta_{k}:=\beta_{k}(X)$ of a topological space $X$ as the number of $k$-dimensional "holes" in $X$, where a $k$-dimensional hole can be thought of as anything that can be continuously transformed into a $k$-dimensional sphere. The zeroth Betti number, $\beta_{0}(X)$, is merely the number of connected components in $X$.

\subsection{Cech complexes and the distance function}

The Cech complex generated by a set of points $\mathcal{P}$ is a simplicial complex, made up of vertices, edges, triangles, and higher dimensional faces. While its general definition is quite broad, we focus on the following special case. 


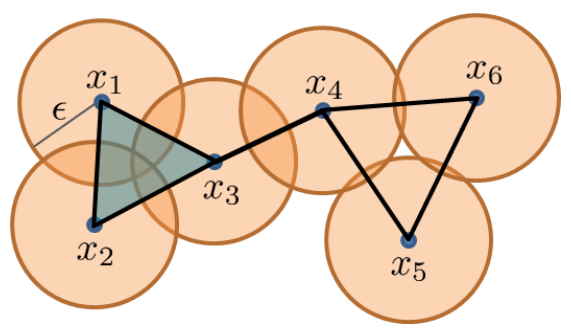

Figure 3: The Čech complex $\check{C}(\mathcal{P}, \epsilon)$, for $\mathcal{P}=\left\{x_{1}, \ldots, x_{6}\right\} \subset \mathbb{R}^{2}$, and some $\epsilon$. The complex contains 6 vertices, 7 edges, and a single 2 -dimensional face.

Definition 4.1 (Čech complex). Let $\mathcal{P}=\left\{x_{1}, x_{2}, \ldots\right\}$ be a collection of points in $\mathbb{R}^{d}$, and let $\epsilon>0$. The Cech complex $\check{C}(\mathcal{P}, \epsilon)$ is constructed as follows:

1. The 0 -simplices (vertices) are the points in $\mathcal{P}$.

2. An $n$-simplex $\left[x_{i_{0}}, \ldots, x_{i_{n}}\right]$ is in $\check{C}(\mathcal{P}, \epsilon)$ if $\bigcap_{k=0}^{n} B_{\epsilon}\left(x_{i_{k}}\right) \neq \emptyset$.

Figure 3 depicts a simple example of a Čech complex in $\mathbb{R}^{2}$.

An important result, known as the "nerve theorem," links Čech complexes $\check{C}(\mathcal{P}, \epsilon)$ and the neighborhood set $\bigcup_{p \in \mathcal{P}} B_{\epsilon}(p)$, and states that they are homotopy equivalent (cf. [9]). Thus, for example, they have the same Betti numbers. Furthermore, both are linked to sublevel sets of the distance function, since it is immediate from the definitions that

$$
d_{\mathcal{P}}^{-1}([0, \epsilon])=\left\{x \in \mathbb{R}^{d}: d_{\mathcal{P}}(x) \leqslant \epsilon\right\}=\bigcup_{p \in \mathcal{P}} B_{\epsilon}(p) \simeq \check{C}(\mathcal{P}, \epsilon) .
$$

\subsection{Critical points and Betti numbers}

Classical Morse theory, in particular the version developed in [11] that applies to the distance function, tells us that, in view of the equivalences in (4.1), there is a connection between the critical points of $d_{\mathcal{P}}$ over the set $d_{\mathcal{P}}^{-1}([0, \epsilon])$, along with their indices, and the Betti numbers of $\check{C}(\mathcal{P}, \epsilon)$. As usual, $\mathcal{P}$ is a point set in $\mathbb{R}^{d}$, and assume that $\mathcal{P}$ is in general position. Then for every critical point of $d_{\mathcal{P}}$ at height $\epsilon$ and of index $k$, for all small enough $\eta$, either

$$
\beta_{k}(\check{C}(\mathcal{P}, \epsilon+\eta))=\beta_{k}(\check{C}(\mathcal{P}, \epsilon-\eta))+1,
$$

or

$$
\beta_{k-1}(\check{C}(\mathcal{P}, \epsilon+\eta))=\beta_{k-1}(\check{C}(\mathcal{P}, \epsilon-\eta))-1 .
$$

Despite this connection, Betti numbers, dealing, as they do, with "holes," are typically determined by global phenomena, and this makes them hard to study directly in the random setting. On the other hand, the structure of critical points is a local phenomenon, which is why, in the random case, we can say more about critical points than what is known for Betti numbers to date. 


\subsection{Random Čech complexes}

Retaining the notation of the previous section, and defining $\beta_{k, n}:=\beta_{k}\left(\check{C}\left(\mathcal{P}_{n}, r_{n}\right)\right)$, our aim will be to examine relationships between the random variables $N_{k, n}$ and the $\beta_{k, n}$ and $\beta_{k-1, n}$. In addition, we shall compare our results for $N_{k, n}$ to those of [16] and [17] for $\beta_{k, n}$, using Morse theory to explain the connections. Note that the results in [16] are phrased in terms of the random samples case (with $n$ i.i.d. points); however, the proofs there can be easily adjusted to fit the Poisson case as well (as in [17] or [6], where both the Poisson and random samples cases are treated).

In direct analogy to the results of Section $3,[\mathbf{1 6}, \mathbf{1 7}]$ show that the limiting behavior of $\check{C}\left(\mathcal{P}_{n}, r_{n}\right)$ splits into three main regimes, depending on the limit of $n r_{n}^{d}$. In the subcritical $\left(n r_{n}^{d} \rightarrow 0\right)$ or dust phase, in which the Cech complex consists mostly of small disconnected particles and very few holes, Theorem 3.2 in [16] states that for $1 \leqslant k \leqslant d-1$,

$$
\lim _{n \rightarrow \infty}\left(n^{k+2} r_{n}^{d(k+1)}\right)^{-1} \mathbb{E}\left\{\beta_{k, n}\right\}=D_{k},
$$

for some constant $D_{k}$ defined in an integral form and related to the $\mu_{k}$ of our Theorem 3.1. In [17] the subcritical phase is explored in more detail, and limit theorems analogous to those of Theorem 3.3 are proved. Combining their results with those in Section 3.1, observe that the $N_{k, n}$ and the $\beta_{k-1, n}$ exhibit similar limiting behavior, and are $O\left(n^{k+1} r_{n}^{d k}\right)$. Furthermore, based on the expected values, we can informally summarize the relationship between the different $N_{k, n}$ and $\beta_{k, n}$ as follows:

$$
\begin{aligned}
& N_{1, n} \gg N_{2, n} \gg N_{3, n} \gg \cdots \gg \quad N_{k_{c}, n} \\
& \approx \quad \approx \quad \approx \\
& \beta_{1, n} \gg \beta_{2, n} \gg \cdots \gg \beta_{k_{c}-1, n},
\end{aligned}
$$

where by $a_{n} \approx b_{n}$ we mean that $a_{n} / b_{n} \rightarrow c \in(0, \infty)$ and by $a_{n} \gg b_{n}$ we mean that $a_{n} / b_{n} \rightarrow \infty$, and $k_{c}$ is as in (3.3). For $k>k_{c}$ all terms are zero with high probability that grows with $k$.

Recall that Morse theory tells us that each critical point of index $k$ contributes either +1 to $\beta_{k, n}$ or -1 to $\beta_{k-1, n}$ (see (4.2), (4.3)). Splitting $N_{k, n}$ accordingly as $N_{k, n}=N_{k, n}^{+}+N_{k, n}^{-}$, the diagram (4.4) implies that $N_{k, n}^{-} \gg N_{k, n}^{+}$. In other words, most of the critical points of index $k$ destroy homology generators rather than create new ones.

For the other regimes, making statements about the Čech complex becomes extremely difficult, and thus the theory is still incomplete.

In the critical phase $\left(n r_{n}^{d} \rightarrow \lambda \in(0, \infty)\right)$, the Čech complex starts to connect and the topology becomes more complex. In addition, once $\lambda$ passes a certain threshold, a giant component emerges (cf. Chapter 9 of $[\mathbf{2 3}]$ ), from which comes the alternate description of this phase as the "percolation phase." Theorem 4.1 in [16] states that for $1 \leqslant k \leqslant d-1$,

$$
\lim _{n \rightarrow \infty} n^{-1} \mathbb{E}\left\{\beta_{k, n}\right\} \in(0, \infty),
$$

although the exact limit is not computed. This agrees with the results in Section 3.2 of this paper. The main difference between the two sets of results is that for critical points we are able to give a closed form expression for the limit mean of $N_{k, n}$ (Theorem 3.5), as well as stronger limit results (Theorems 3.6, 3.7). This will be useful below, when we discuss Euler characteristics. 
In the supercritical regime $\left(n r_{n}^{d} \rightarrow \infty\right)$ even less is known about the Čech complex. In general, the Čech complex becomes highly connected, the topology becomes simpler, and the Betti numbers decrease. Theorem 6.1 of $[\mathbf{1 6}]$ gives the precise results that if $f$ is a uniform density with a compact and convex support, and $\lim _{n \rightarrow \infty}(\log n / n)^{-1 / d} r_{n}>0$, then

$$
\lim _{n \rightarrow \infty} \mathbb{P}\left(\beta_{0, n}=1, \beta_{1, n}=\cdots=\beta_{d-1, n}=0\right)=1,
$$

which is described in [16] by saying that $\check{C}\left(\mathcal{P}_{n}, r_{n}\right)$ is "asymptotically almost surely contractible." We have no analogous result about critical points, nor could we, since $N_{k, n}$ is $O(n)$ and thus $N_{k, n} \rightarrow \infty$ (Section 3.2). However, Corollary 4.2 below gives information about the Euler characteristic of the Čech complex which is different from, but related to, (4.5). (Note that (4.5) requires that the underlying probability density is lower bounded with convex support, the same assumption we adopted in Section 3.2.)

To conclude this section, we present a novel statement about the Čech complex $\check{C}\left(\mathcal{P}_{n}, r_{n}\right)$ that can be made based on the results in Section 3. The Euler characteristic of a simplicial complex $\mathcal{S}$ has a number of equivalent definitions, and a number of important applications. One of the definitions, via Betti numbers, is

$$
\chi(\mathcal{S})=\sum_{k=0}^{\infty}(-1)^{k} \beta_{k}(\mathcal{S}) .
$$

However, $\chi(\mathcal{S})$ also has a definition via indices of critical points of appropriately defined functions supported on $\mathcal{S}$, and this leads to

Corollary 4.2. Let $\chi_{n}$ be the Euler characteristic of $\check{C}\left(\mathcal{P}_{n}, r_{n}\right)$. Then

$$
\lim _{n \rightarrow \infty} n^{-1} \mathbb{E}\left\{\chi_{n}\right\}= \begin{cases}1 & n r_{n}^{d} \rightarrow 0 \\ 1+\sum_{k=1}^{d}(-1)^{k} \gamma_{k}(\lambda) & n r_{n}^{d} \rightarrow \lambda \in(0, \infty), \\ 0 & n r_{n}^{d} \rightarrow \infty\end{cases}
$$

Moreover, when $n r_{n}^{d} \rightarrow \infty$ and $n r_{n}^{d} \geqslant D^{\star} \log n$ (with $D^{\star}$ as in Proposition 3.9), then $\mathbb{E}\left\{\chi_{n}\right\} \rightarrow 1$.

The proof of Corollary 4.2 is presented in Section 9. Note that (4.7) cannot be proven using only the existing results on Betti numbers, since the values of the limiting mean in the critical and supercritical regimes are not available. This demonstrates one of the advantages of studying the homology of the Čech complex via the distance function.

In closing we note some of the implications of Corollary 4.2. In the subcritical phase, we have that $\chi_{n} \sim n$, which agrees with the intuition developed so far that, in this range, the Cech complex consists of mostly small disconnected particles and very few holes. In the critical range we have a non-trivial limit resulting from the fact that the Čech complex has many holes of all possible dimensions. In the supercritical range, $\chi_{n} \sim 1$, which is exactly what we get when $\beta_{0, n}=1, \beta_{1, n}=\cdots=\beta_{d-1, n}=0$ (cf. (4.6), (4.5)). Since $n^{-1} \mathbb{E}\left\{\chi_{n}\right\} \rightarrow 0$ in this regime, it is clear now why the numerics of Figure 2 showed that $\sum_{k=0}^{3}(-1)^{k} \gamma_{k}(\infty) \approx 0$. Finally, note that in a sequel [8], we explore the Čech complex when the samples are generated by a distribution supported 
on a closed manifold $\mathcal{M}$. In this case we can make a much more concrete statement, and prove that $\beta_{k, n} \rightarrow \beta_{k}(\mathcal{M})$ with an appropriate choice of radius $r_{n}$. A different direction, in which the underlying samples are generated by stochastic processes with dependence between the points, can be found in $[\mathbf{2 6}]$.

\section{Some notation and elementary considerations}

The remaining sections of the paper are devoted to proofs of the results in Sections 3 and 4, and are organized according to situations: sub-critical (dust), critical (percolation), and super-critical. In this section we list some common notation and note some simple facts that will be used in many of them.

- Henceforth, $k$ will be fixed, and whenever we use $\mathcal{Y}, \mathcal{Y}^{\prime}$, or $\mathcal{Y}_{i}$ we implicitly assume that $|\mathcal{Y}|=\left|\mathcal{Y}^{\prime}\right|=\left|\mathcal{Y}_{i}\right|=k+1$, unless stated otherwise.

- Usually, finite subsets of $\mathbb{R}^{d}$ will be denoted calligraphically $(\mathcal{X}, \mathcal{Y})$. However, inside integrals we use boldfacing and lowercase $(\mathbf{x}, \mathbf{y})$.

- For $x \in \mathbb{R}^{d}, \mathbf{x} \in\left(\mathbb{R}^{d}\right)^{k+1}$, and $\mathbf{y} \in\left(\mathbb{R}^{d}\right)^{k}$, we use the shorthand

$$
\begin{aligned}
f(\mathbf{x}) & :=f\left(x_{1}\right) f\left(x_{2}\right) \cdots f\left(x_{k+1}\right), \\
f\left(x+r_{n} \mathbf{y}\right) & :=f\left(x+r_{n} y_{1}\right) f\left(x+r_{n} y_{2}\right) \cdots f\left(x+r_{n} y_{k}\right), \\
h(0, \mathbf{y}) & :=h\left(0, y_{1}, \ldots, y_{k}\right) .
\end{aligned}
$$

- The symbol " $c$ " denotes a constant value, which might depend on $d$ (ambient dimension), $f$ (the probability density of the samples), and $k$ (the Morse index), but on neither $n$ nor $r_{n}$. The actual value of $c^{\star}$ may change between and even within lines.

- While not exactly a notational issue, we shall often use the fact that, for every $k$, $n^{-k}\left(\begin{array}{l}n \\ k\end{array}\right) \rightarrow 1 / k !$ as $n \rightarrow \infty$, and there is a $c^{\star}$ such that $\left(\begin{array}{l}n \\ k\end{array}\right) \leqslant c^{\star} n^{k}$.

Lemma 5.1. Let $\mathcal{X}=\left(X_{1}, \ldots, X_{k}\right)$ be a set of $k$ i.i.d. points in $\mathbb{R}^{d}$ sampled from a bounded density $f$. Then there exists a constant $c^{\star}$ such that

$$
\mathbb{P}(\mathcal{X} \text { is contained in a ball with radius } r) \leqslant c^{\star} r^{d(k-1)} .
$$

Proof. If $\mathcal{X}$ is bounded by a ball with radius $r$, then $X_{2}, \ldots, X_{k}$ are all within distance $2 r$ from $X_{1}$; thus

$$
\begin{aligned}
\mathbb{P}(\mathcal{X} \text { is bounded by a ball of radius } r) & \leqslant \int_{\mathbb{R}^{d}}\left(\int_{B_{2 r}(x)} f(y) d y\right)^{k-1} f(x) d x \\
& \leqslant \int_{\mathbb{R}^{d}}\left(f_{\max } \operatorname{Vol}\left(B_{2 r}(x)\right)\right)^{k-1} f(x) d x \\
& =f_{\max }^{k-1} \omega_{d}^{k-1}(2 r)^{d(k-1)} \\
& :=c^{\star} r^{d(k-1)},
\end{aligned}
$$

where $f_{\text {max }}:=\sup _{x \in \mathbb{R}^{d}} f(x)$, and $\omega_{d}$ is the volume of the unit ball in $\mathbb{R}^{d}$. 


\section{Means for the subcritical range $\left(n r_{n}^{d} \rightarrow 0\right)$}

We start by proving Theorem 3.1 (the limit expectation), which requires the following important lemma. Note that the lemma has two implications. Firstly, it gives a precise order of magnitude, with constant, for the probability that $k+1$ points in the $r_{n}$-neighborhood of a point in $\mathcal{X}_{n}$ generate an index- $k$ critical point. Secondly, it implies that if an additional, high density set of Poisson points is added to the picture, the probability that any of these will be in the ball containing the $k+1$ original points is of a smaller order of magnitude.

Lemma 6.1. Let $\mathcal{Y} \subset \mathcal{X}_{n}$ be a set of $k+1$ random i.i.d. points with density function $f$, independent of the Poisson process $\mathcal{P}_{n}$. Then,

$$
\lim _{n \rightarrow \infty} r_{n}^{-d k} \mathbb{E}\left\{h_{r_{n}}(\mathcal{Y})\right\}=\lim _{n \rightarrow \infty} r_{n}^{-d k} \mathbb{E}\left\{g_{r_{n}}\left(\mathcal{Y}, \mathcal{Y} \cup \mathcal{P}_{n}\right)\right\}=(k+1) ! \mu_{k}
$$

Proof. Note that from the definition of $h_{\epsilon}(\cdot)$, it follows that

$$
h_{\epsilon}(x, x+\epsilon \mathbf{y}):=h_{\epsilon}\left(x, x+\epsilon y_{1}, \ldots, x+\epsilon y_{k}\right)=h_{1}(0, \mathbf{y}) .
$$

Thus, using the change of variables $\mathbf{x} \rightarrow\left(x, x+r_{n} \mathbf{y}\right)$,

$$
\begin{aligned}
\mathbb{E}\left\{h_{r_{n}}(\mathcal{Y})\right\} & =\int_{\left(\mathbb{R}^{d}\right)^{k+1}} f(\mathbf{x}) h_{r_{n}}(\mathbf{x}) d \mathbf{x} \\
& =r_{n}^{d k} \int_{\mathbb{R}^{d}} \int_{\left(\mathbb{R}^{d}\right)^{k}} f(x) f\left(x+r_{n} \mathbf{y}\right) h_{r_{n}}\left(x, x+r_{n} \mathbf{y}\right) d \mathbf{y} d x \\
& =r_{n}^{d k} \int_{\mathbb{R}^{d}} f(x) \int_{\left(\mathbb{R}^{d}\right)^{k}} f\left(x+r_{n} \mathbf{y}\right) h_{1}(0, \mathbf{y}) d \mathbf{y} d x
\end{aligned}
$$

Now, for $h_{1}(0, \mathbf{y})$ to be nonzero, all the elements $y_{1}, \ldots, y_{k} \in \mathbb{R}^{d}$ must lie inside $B_{2}(0)$ - the ball of radius 2 around the origin. Therefore,

$$
\left|f\left(x+r_{n} \mathbf{y}\right) h_{1}(0, \mathbf{y})\right| \leqslant f_{\max }^{k} \mathbb{1}_{B_{2}(0)}\left(y_{1}\right) \cdots \mathbb{1}_{B_{2}(0)}\left(y_{k}\right),
$$

and applying the dominated convergence theorem (DCT) to (6.1) yields

$$
\lim _{n \rightarrow \infty} \int_{\left(\mathbb{R}^{d}\right)^{k}} f\left(x+r_{n} \mathbf{y}\right) h_{1}(0, \mathbf{y}) d x d \mathbf{y}=f^{k}(x) \int_{\left(\mathbb{R}^{d}\right)^{k}} h_{1}(0, \mathbf{y}) d \mathbf{y},
$$

from which follows

$$
\lim _{n \rightarrow \infty} r_{n}^{-d k} \mathbb{E}\left\{h_{r_{n}}(\mathcal{Y})\right\}=\int_{\mathbb{R}^{d}} f^{k+1}(x) d x \int_{\left(\mathbb{R}^{d}\right)^{k}} h_{1}(0, \mathbf{y}) d \mathbf{y}=(k+1) ! \mu_{k},
$$

completing the proof for $h_{r_{n}}(\mathcal{Y})$.

Next, the definition of $\mathcal{P}_{n}$ as a Poisson process with intensity $n f(x)$ implies

$$
\mathbb{E}\left\{g_{r_{n}}\left(\mathcal{Y}, \mathcal{Y} \cup \mathcal{P}_{n}\right) \mid \mathcal{Y}\right\}=h_{r_{n}}(\mathcal{Y}) \mathbb{P}\left(B(\mathcal{Y}) \cap \mathcal{P}_{n}=\emptyset \mid \mathcal{Y}\right)=h_{r_{n}}(\mathcal{Y}) e^{-n p(\mathcal{Y})}
$$


Thus,

$$
\begin{aligned}
\mathbb{E}\left\{g_{r_{n}}\left(\mathcal{Y}, \mathcal{Y} \cup \mathcal{P}_{n}\right)\right\} & =\mathbb{E}\left\{\mathbb{E}\left\{g_{r_{n}}\left(\mathcal{Y}, \mathcal{Y} \cup \mathcal{P}_{n}\right) \mid \mathcal{Y}\right\}\right\} \\
& =\int_{\left(\mathbb{R}^{d}\right)^{k+1}} f(\mathbf{x}) h_{r_{n}}(\mathbf{x}) e^{-n p(\mathbf{x})} d \mathbf{x} \\
& =r_{n}^{d k} \int_{\mathbb{R}^{d}} f(x) \int_{\left(\mathbb{R}^{d}\right)^{k}} f\left(x+r_{n} \mathbf{y}\right) h_{1}(0, \mathbf{y}) e^{-n p\left(x, x+r_{n} \mathbf{y}\right)} d \mathbf{y} d x .
\end{aligned}
$$

The integrand here is smaller than or equal to the one in (6.1); therefore we can safely apply the DCT to it. To find the limit, first note that

$$
\begin{aligned}
n p\left(x, x+r_{n} \mathbf{y}\right) & =n \int_{B\left(x, x+r_{n} \mathbf{y}\right)} f(z) d z \\
& =n \operatorname{Vol}\left(B\left(x, x+r_{n} \mathbf{y}\right)\right) \frac{\int_{B\left(x, x+r_{n} \mathbf{y}\right)} f(z) d z}{\operatorname{Vol}\left(B\left(x, x+r_{n} \mathbf{y}\right)\right)} \\
& =n \omega_{d}\left(r_{n} R(0, \mathbf{y})\right)^{d} \frac{\int_{B\left(x, x+r_{n} \mathbf{y}\right)} f(z) d z}{\operatorname{Vol}\left(B\left(x, x+r_{n} \mathbf{y}\right)\right)} .
\end{aligned}
$$

Applying the Lebesgue differentiation theorem yields

$$
\lim _{n \rightarrow \infty} \frac{\int_{B\left(x, x+r_{n} \mathbf{y}\right)} f(z) d z}{\operatorname{Vol}\left(B\left(x, x+r_{n} \mathbf{y}\right)\right)}=f(x) .
$$

Therefore, since $n r_{n}^{d} \rightarrow 0$, we have

$$
\lim _{n \rightarrow \infty} n p\left(x, x+r_{n} \mathbf{y}\right)=0 .
$$

Thus, we have

$$
\lim _{n \rightarrow \infty} r_{n}^{-d k} \mathbb{E}\left\{g_{r_{n}}\left(\mathcal{Y}, \mathcal{P}_{n}\right)\right\}=\lim _{n \rightarrow \infty} r_{n}^{-d k} \mathbb{E}\left\{h_{r_{n}}(\mathcal{Y})\right\}=(k+1) ! \mu_{k},
$$

and we are done.

Using the previous lemma, it is now easy to prove Theorem 3.1.

Proof of Theorem 3.1. Note that $N_{k, n}=\sum_{\mathcal{Y} \subset \mathcal{P}_{n}} g_{r_{n}}\left(\mathcal{Y}, \mathcal{P}_{n}\right)$. Applying Theorem B.1 therefore yields that

$$
\mathbb{E}\left\{N_{k, n}\right\}=\frac{n^{k+1}}{(k+1) !} \mathbb{E}\left\{g_{r_{n}}\left(\mathcal{Y}^{\prime}, \mathcal{Y}^{\prime} \cup \mathcal{P}_{n}\right)\right\}
$$

where $\mathcal{Y}^{\prime}$ is a copy of $\mathcal{Y}$ independent of $\mathcal{P}_{n}$. Lemma 6.1 then implies

$$
\lim _{n \rightarrow \infty}\left(n^{k+1} r_{n}^{d k}\right)^{-1} \mathbb{E}\left\{N_{k, n}\right\}=\mu_{k},
$$

as required.

\section{Variances and limit distributions for the subcritical range}

The proofs of Theorems 3.2 and 3.3 split into three different cases, depending on the limit of $n^{k+1} r_{n}^{d k}$. 
Case 1: $n^{k+1} r_{n}^{d k} \rightarrow 0$

We start with the limit variance for this case.

Proof of Theorem 3.2. We start by writing

$$
\begin{aligned}
\mathbb{E}\left\{N_{k, n}^{2}\right\} & =\mathbb{E}\left\{\sum_{\mathcal{Y}_{1} \subset \mathcal{P}_{n}} \sum_{\mathcal{Y}_{2} \subset \mathcal{P}_{n}} g_{r_{n}}\left(\mathcal{Y}_{1}, \mathcal{P}_{n}\right) g_{r_{n}}\left(\mathcal{Y}_{2}, \mathcal{P}_{n}\right)\right\} \\
& =\sum_{j=0}^{k+1} \mathbb{E}\left\{\sum_{\mathcal{Y}_{1} \subset \mathcal{P}_{n}} \sum_{\mathcal{Y}_{2} \subset \mathcal{P}_{n}} g_{r_{n}}\left(\mathcal{Y}_{1}, \mathcal{P}_{n}\right) g_{r_{n}}\left(\mathcal{Y}_{2}, \mathcal{P}_{n}\right) \mathbb{1}\left\{\left|\mathcal{Y}_{1} \cap \mathcal{Y}_{2}\right|=j\right\}\right\} \\
& :=\sum_{j=0}^{k+1} \mathbb{E}\left\{I_{j}\right\} .
\end{aligned}
$$

Note that

$$
I_{k+1}=\sum_{\mathcal{Y}_{1} \subset \mathcal{P}_{n}} g_{r_{n}}\left(\mathcal{Y}_{1}, \mathcal{P}_{n}\right)=N_{k, n}
$$

Thus, from Theorem 3.1,

$$
\lim _{n \rightarrow \infty}\left(n^{k+1} r_{n}^{d k}\right)^{-1} \mathbb{E}\left\{I_{k+1}\right\}=\mu_{k} .
$$

Next, for $0 \leqslant j<k+1$, using Corollary B.2 we have

$$
\mathbb{E}\left\{I_{j}\right\}=c^{\star} n^{2 k+2-j} \mathbb{E}\left\{g_{r_{n}}\left(\mathcal{Y}_{1}^{\prime}, \mathcal{Y}_{12}^{\prime} \cup \mathcal{P}_{n}\right) g_{r_{n}}\left(\mathcal{Y}_{2}^{\prime}, \mathcal{Y}_{12}^{\prime} \cup \mathcal{P}_{n}\right)\right\}_{\left|\mathcal{Y}_{1}^{\prime} \cap \mathcal{Y}_{2}^{\prime}\right|=j},
$$

where $\mathcal{Y}_{12}^{\prime}=\mathcal{Y}_{1}^{\prime} \cup \mathcal{Y}_{2}^{\prime}$ is a set of $2 k-j$ i.i.d. points in $\mathbb{R}^{d}$ with density $f(x)$, independent of $\mathcal{P}_{n}$, and $\left|\mathcal{Y}_{1}^{\prime} \cap \mathcal{Y}_{2}^{\prime}\right|=j$.

For $0<j<k+1$, if $\left|\mathcal{Y}_{1} \cap \mathcal{Y}_{2}\right|=j$ and $g_{r_{n}}\left(\mathcal{Y}_{1}^{\prime}, \mathcal{Y}_{12}^{\prime} \cup \mathcal{P}_{n}\right) g_{r_{n}}\left(\mathcal{Y}_{2}^{\prime}, \mathcal{Y}_{12}^{\prime} \cup \mathcal{P}_{n}\right)=1$, then necessarily the $2 k+2-j$ points in $\mathcal{Y}_{1}^{\prime} \cup \mathcal{Y}_{2}^{\prime}$ are bounded by a ball of radius $2 r_{n}$, and using Lemma 5.1 we have

$$
\mathbb{E}\left\{I_{j}\right\}=\leqslant c^{\star} n^{2 k+2-j} r_{n}^{d(2 k+1-j)} .
$$

Thus,

$$
\left(n^{k+1} r_{n}^{d k}\right)^{-1} \mathbb{E}\left\{I_{j}\right\} \leqslant c^{\star}\left(n r_{n}^{d}\right)^{k+1-j} \rightarrow 0 .
$$

For $j=0$, the sets $\mathcal{Y}_{1}^{\prime}$ and $\mathcal{Y}_{2}^{\prime}$ are independent. Since $g_{r_{n}}\left(\mathcal{Y}_{i}^{\prime}, \mathcal{Y}_{12}^{\prime} \cup \mathcal{P}_{n}\right) \leqslant h_{r_{n}}\left(\mathcal{Y}_{i}^{\prime}\right)$, we have

$$
\mathbb{E}\left\{g_{r_{n}}\left(\mathcal{Y}_{1}^{\prime}, \mathcal{Y}_{12}^{\prime} \cup \mathcal{P}_{n}\right) g_{r_{n}}\left(\mathcal{Y}_{2}^{\prime}, \mathcal{Y}_{12}^{\prime} \cup \mathcal{P}_{n}\right)\right\} \leqslant \mathbb{E}\left\{h_{r_{n}}\left(\mathcal{Y}_{1}^{\prime}\right) h_{r_{n}}\left(\mathcal{Y}_{2}^{\prime}\right)\right\}=\left(\mathbb{E}\left\{h_{r_{n}}\left(\mathcal{Y}_{1}^{\prime}\right)\right\}\right)^{2} .
$$

Therefore,

$$
\mathbb{E}\left\{I_{0}\right\} \leqslant c^{\star} n^{2(k+1)}\left(\mathbb{E}\left\{h_{r_{n}}(\mathcal{Y})\right\}\right)^{2} .
$$

Using Lemma 6.1 together with the fact that $n^{k+1} r_{n}^{d k} \rightarrow 0$ yields

$$
\left(n^{k+1} r_{n}^{d k}\right)^{-1} \mathbb{E}\left\{I_{0}\right\} \leqslant c^{\star} n^{k+1} r_{n}^{d k}\left(r_{n}^{-d k} \mathbb{E}\left\{h_{r_{n}}(\mathcal{Y})\right\}\right)^{2} \rightarrow 0 .
$$

Combining (7.2), (7.3), and (7.4) yields

$$
\lim _{n \rightarrow \infty}\left(n^{k+1} r_{n}^{d k}\right)^{-1} \mathbb{E}\left\{N_{k, n}^{2}\right\}=\mu_{k} .
$$


In addition, Theorem 3.1 implies

$$
\left(n^{k+1} r_{n}^{d k}\right)^{-1}\left(\mathbb{E}\left\{N_{k, n}\right\}\right)^{2}=n^{k+1} r_{n}^{d k}\left(\left(n^{k+1} r_{n}^{d k}\right)^{-1} \mathbb{E}\left\{N_{k, n}\right\}\right)^{2} \rightarrow 0 .
$$

Therefore, since $\operatorname{var}\left\{N_{k, n}\right\}=\mathbb{E} N_{k, n}^{2}-\left(\mathbb{E} N_{k, n}\right)^{2}$, we conclude that

$$
\lim _{n \rightarrow \infty}\left(n^{k+1} r_{n}^{d k}\right)^{-1} \operatorname{Var}\left(N_{k, n}\right)=\mu_{k},
$$

which completes the proof.

Next, we wish to prove the first part of Theorem 3.3, i.e., that $N_{k, n} \stackrel{L_{2}}{\rightarrow} 0$.

Proof of Theorem 3.3-Part 1. Clearly, it suffices to show that

$$
\lim _{n \rightarrow \infty} \mathbb{E}\left\{N_{k, n}^{2}\right\}=0 \text {. }
$$

However, in the previous proof, we saw that

$$
\lim _{n \rightarrow \infty}\left(n^{k+1} r_{n}^{d k}\right)^{-1} \mathbb{E}\left\{N_{k, n}^{2}\right\}=\mu_{k}
$$

Since $n^{k+1} r_{n}^{d k} \rightarrow 0,(7.5)$ follows immediately, and we are done.

Case 2: $n^{k+1} r_{n}^{d k} \rightarrow \alpha \in(0, \infty)$

Proof of Theorem 3.2 continued. The proof in this case is similar to the previous one. We define $I_{j}$ the same way as in (7.1). The same arguments that led to (7.2) and (7.3) can be repeated here, providing the limits of $\mathbb{E}\left\{I_{j}\right\}$ for $0<j \leqslant k+1$. The only difference is in how to bound the term $\mathbb{E}\left\{I_{0}\right\}$. For that, a proof in the spirit of Lemma 6.1 can be used to show that

$$
\lim _{n \rightarrow \infty} r_{n}^{-2 d k} \mathbb{E}\left\{g_{r_{n}}\left(\mathcal{Y}_{1}^{\prime}, \mathcal{Y}_{12}^{\prime} \cup \mathcal{P}_{n}\right) g_{r_{n}}\left(\mathcal{Y}_{2}^{\prime}, \mathcal{Y}_{12}^{\prime} \cup \mathcal{P}_{n}\right)\right\}_{\left|\mathcal{Y}_{1}^{\prime} \cap \mathcal{Y}_{2}^{\prime}\right|=0}=\left((k+1) ! \mu_{k}\right)^{2}
$$

Using Corollary B.2, we have

$$
\begin{aligned}
& \lim _{n \rightarrow \infty}\left(n^{k+1} r_{n}^{d k}\right)^{-1} \mathbb{E}\left\{I_{0}\right\} \\
& \quad=\lim _{n \rightarrow \infty}\left(n^{k+1} r_{n}^{d k}\right)^{-1} \frac{n^{2 k+2}}{((k+1) !)^{2}} \mathbb{E}\left\{g_{r_{n}}\left(\mathcal{Y}_{1}^{\prime}, \mathcal{Y}_{12}^{\prime} \cup \mathcal{P}_{n}\right) g_{r_{n}}\left(\mathcal{Y}_{2}^{\prime}, \mathcal{Y}_{12}^{\prime} \cup \mathcal{P}_{n}\right)\right\}_{\left|\mathcal{Y}_{1}^{\prime} \cap \mathcal{Y}_{2}^{\prime}\right|=0},
\end{aligned}
$$

and therefore,

$$
\lim _{n \rightarrow \infty}\left(n^{k+1} r_{n}^{d k}\right)^{-1} \mathbb{E}\left\{I_{0}\right\}=\alpha \mu_{k}^{2}
$$

Finally, we also have

$$
\lim _{n \rightarrow \infty}\left(n^{k+1} r_{n}^{d k}\right)^{-1}\left(\mathbb{E}\left\{N_{k, n}\right\}\right)^{2}=\alpha \mu_{k}^{2} .
$$

This completes the proof.

For $X, Y$ random variables taking values in $\mathbb{N}$, define the total variation distance to be $d_{\mathrm{TV}}(X, Y):=\sup _{A \subset \mathbb{N}}|\mathbb{P}(X \in A)-\mathbb{P}(Y \in A)|$. To prove the Poisson limit of Theorem 3.3, we need the following lemma.

Lemma 7.1. Let $S_{k, n}:=\sum_{\mathcal{Y} \subset \mathcal{P}_{n}} h_{r_{n}}(\mathcal{Y})$, and let $Z \sim$ Poisson $\left(\mathbb{E}\left\{S_{k, n}\right\}\right)$. If $n r_{n}^{d} \rightarrow 0$, then

$$
d_{\mathrm{TV}}\left(S_{k, n}, Z\right) \leqslant c^{\star} n r_{n}^{d}
$$


Proof. The proof is very similar to the proof of Theorem 3.4 in [23], and uses the Poisson approximation given in Theorem C.2. Let $A \subset \mathbb{N}$ be a set of natural numbers, we wish to bound the difference

$$
\left|\mathbb{P}\left(S_{k, n} \in A\right)-\mathbb{P}(Z \in A)\right| .
$$

Start by conditioning on $\left|\mathcal{P}_{n}\right|$, the number of points in $\mathcal{P}_{n}$.

$$
\begin{aligned}
& \left|\mathbb{P}\left(S_{k, n} \in A\right)-\mathbb{P}(Z \in A)\right| \\
& \quad=\left|\sum_{m=0}^{\infty}\left(\mathbb{P}\left(S_{k, n} \in A|| \mathcal{P}_{n} \mid=m\right)-\mathbb{P}(Z \in A)\right) \mathbb{P}\left(\left|\mathcal{P}_{n}\right|=m\right)\right| \\
& \quad \leqslant \sum_{m=0}^{\infty}\left|\mathbb{P}\left(S_{k, n} \in A|| \mathcal{P}_{n} \mid=m\right)-\mathbb{P}(Z \in A)\right| \mathbb{P}\left(\left|\mathcal{P}_{n}\right|=m\right) .
\end{aligned}
$$

Given $\left|\mathcal{P}_{n}\right|=m$, let $\mathcal{I}_{m}=\{\mathbf{i} \subset\{1,2, \ldots, m\}:|\mathbf{i}|=k+1\}$. Then, for $\mathbf{i}=\left\{i_{0}, \ldots, i_{k}\right\}$ and $\mathcal{X}_{\mathbf{i}}=\left\{X_{i_{0}}, \ldots, X_{i_{k}}\right\}$, we can write

$$
S_{k, n}=\sum_{\mathbf{i} \in \mathcal{I}_{m}} h_{r_{n}}\left(\mathcal{X}_{\mathbf{i}}\right)
$$

Set $\mathcal{N}_{\mathbf{i}}=\left\{\mathbf{j} \in \mathcal{I}_{m}:|\mathbf{i} \cap \mathbf{j}|>0\right\}$, and let $\sim$ be a relation on $\mathcal{I}_{m}$ such that $\mathbf{i} \sim \mathbf{j}$ if and only if $\mathbf{j} \in \mathcal{N}_{i}$. For $\mathbf{i} \neq \mathbf{j}, \mathcal{X}_{\mathbf{i}}$ and $\mathcal{X}_{\mathbf{j}}$ are independent unless $\mathbf{j} \in \mathcal{N}_{\mathbf{i}}$. Thus, the graph $\left(\mathcal{I}_{m}, \sim\right)$ is the dependency graph for $\xi_{\mathbf{i}}:=h_{r_{n}}\left(\mathcal{X}_{\mathbf{i}}\right)$.

Now, if $h_{r_{n}}\left(\mathcal{X}_{\mathbf{i}}\right) \neq 0$, then the $k+1$ points in $\mathcal{X}_{\mathbf{i}}$ are bounded by a ball of radius $r_{n}$, and using Lemma 5.1 we have

$$
p_{\mathbf{i}}:=\mathbb{E}\left\{\xi_{\mathbf{i}}\right\} \leqslant c^{\star} r_{n}^{d k}
$$

Therefore,

$$
\sum_{\mathbf{i} \in \mathcal{I}_{m}} \sum_{\mathbf{j} \in \mathcal{N}_{\mathbf{i}}} p_{\mathbf{i}} p_{\mathbf{j}} \leqslant\left(\begin{array}{c}
m \\
k+1
\end{array}\right)\left(\left(\begin{array}{c}
m \\
k+1
\end{array}\right)-\left(\begin{array}{c}
m-k-1 \\
k+1
\end{array}\right)\right) c^{\star} r_{n}^{2 d k} \leqslant c^{\star} m^{2 k+1} r_{n}^{2 d k}
$$

Next, if $\mathbf{i} \sim \mathbf{j}$ with $|\mathbf{i} \cap \mathbf{j}|=l>0$, and $h_{r_{n}}\left(\mathcal{X}_{\mathbf{i}}\right) h_{r_{n}}\left(\mathcal{X}_{\mathbf{j}}\right) \neq 0$, then necessarily the $2 k+$ $2-l$ points in $\mathcal{X}_{\mathbf{i}} \cup \mathcal{X}_{\mathbf{j}}$ are bounded by a ball of radius $2 r_{n}$, and therefore,

$$
p_{\mathbf{i}, \mathbf{j}}:=\mathbb{E}\left\{\xi_{\mathbf{i}} \xi_{\mathbf{j}}\right\} \leqslant c^{\star} r_{n}^{d(2 k+1-l)}
$$

Thus,

$$
\begin{aligned}
\sum_{\mathbf{i} \in \mathcal{I}_{m}} \sum_{\mathbf{j} \in \mathcal{N}_{\mathbf{i}} \backslash\{\mathbf{i}\}} p_{\mathbf{i}, \mathbf{j}} & \leqslant \sum_{l=1}^{k}\left(\begin{array}{c}
m \\
k+1
\end{array}\right)\left(\begin{array}{c}
m-k-1 \\
k+1-l
\end{array}\right)\left(\begin{array}{c}
k+1 \\
l
\end{array}\right) c^{\star} r_{n}^{d(2 k+1-l)} \\
& \leqslant c^{\star} \sum_{l=1}^{k} m^{2 k+2-l} r_{n}^{d(2 k+1-l)} .
\end{aligned}
$$

Finally, using Lemma 6.1 it is easy to prove that

$$
\lim _{n \rightarrow \infty}\left(n^{k+1} r_{n}^{d k}\right)^{-1} \mathbb{E}\left\{S_{k, n}\right\}=\mu_{k},
$$


which implies that

$$
\frac{1}{\mathbb{E}\left\{S_{k, n}\right\}} \leqslant c^{\star}\left(n^{k+1} r_{n}^{d k}\right)^{-1} .
$$

Therefore, from Theorem C.2, we can conclude that

$$
\left|\mathbb{P}\left(S_{k, n} \in A|| \mathcal{P}_{n} \mid=m\right)-\mathbb{P}(Z \in A)\right| \leqslant c^{\star} n^{-(k+1)} \sum_{l=1}^{k} m^{2 k+2-l} r_{n}^{d(k+1-l)} .
$$

Substituting back into (7.6), we have

$$
d_{\mathrm{TV}}\left(S_{k, n}, Z\right) \leqslant c^{\star} n^{-k+1} \sum_{l=1}^{k} r_{n}^{d(k+1-l)} \mathbb{E}\left\{\left|\mathcal{P}_{n}\right|^{2 k+2-l}\right\} .
$$

Since $\left|\mathcal{P}_{n}\right| \sim$ Poisson $(n)$, it is easy to find a constant $c^{\star}$ such that

$$
\mathbb{E}\left\{\left|\mathcal{P}_{n}\right|^{2 k+2-l}\right\} \leqslant c^{\star} n^{2 k+2-l},
$$

for every $1 \leqslant l \leqslant k$. So, finally, we have that

$$
d_{\mathrm{TV}}\left(S_{k, n}, Z\right) \leqslant c^{\star} \sum_{l=1}^{k} n^{k+1-l} r_{n}^{d(k+1-l)} \leqslant c^{\star} n r_{n}^{d},
$$

since $n r_{n}^{d} \rightarrow 0$ and so is bounded.

Note that the previous result did not use the assumption that $n^{k+1} r_{n}^{d k} \rightarrow \alpha \in$ $(0, \infty)$. However, to prove an analogous result for $N_{k, n}$ rather than $S_{k, n}$, we shall need it. We shall also need the following two lemmas, the second of which follows easily from the first, which itself follows from a simple calculation.

Lemma 7.2. Let $X, Y$ be integer random variables defined over the same probability space, such that $\Delta:=X-Y \geqslant 0$. Then $d_{\mathrm{TV}}(X, Y) \leqslant \mathbb{E}\{\Delta\}$.

Lemma 7.3. Let $X \sim \operatorname{Poisson}\left(\lambda_{x}\right), Y \sim \operatorname{Poisson}\left(\lambda_{y}\right)$. Then $d_{\mathrm{TV}}(X, Y) \leqslant\left|\lambda_{x}-\lambda_{y}\right|$.

Proof of Theorem 3.3-Part 2. For a start, we need to prove that $d_{\mathrm{TV}}\left(N_{k, n}, S_{k, n}\right) \leqslant$ $c^{\star} n r_{n}^{d}$. To this end, define $\Delta:=S_{k, n}-N_{k, n}$ and note that $\Delta$ counts the number of subsets $\mathcal{Y} \subset \mathcal{P}_{n}$ for which $h_{r_{n}}(\mathcal{Y})=1$ but $g_{r_{n}}\left(\mathcal{Y}, \mathcal{P}_{n}\right)=0$. This implies that there exists $X \in \mathcal{P}_{n} \backslash \mathcal{Y}$ for which $X \in B(\mathcal{Y})$. Thus, $\Delta$ is bounded from above by $k+2$ times the number of $(k+2)$-subsets contained in a ball of radius $r_{n}$. From Lemma 5.1 and Lemma 7.2 we have

$$
d_{\mathrm{TV}}\left(N_{k, n}, S_{k, n}\right) \leqslant \mathbb{E}\{\Delta\} \leqslant c^{\star} n^{k+2} r_{n}^{d(k+1)} \leqslant c^{\star}\left(n^{k+1} r_{n}^{d k}\right)\left(n r_{n}^{d}\right) \leqslant c^{\star}\left(n r_{n}^{d}\right),
$$

where we used the fact that $n^{k+1} r_{n}^{d k}$ is bounded.

Next, if

$$
Z_{N} \sim \operatorname{Poisson}\left(\mathbb{E}\left\{N_{k, n}\right\}\right) \quad \text { and } \quad Z_{S} \sim \operatorname{Poisson}\left(\mathbb{E}\left\{S_{k, n}\right\}\right),
$$


then from Lemma 7.1 and the triangle inequality,

$$
\begin{aligned}
d_{\mathrm{TV}}\left(N_{k, n}, Z_{N}\right) & \leqslant d_{\mathrm{TV}}\left(N_{k, n}, S_{k, n}\right)+d_{\mathrm{TV}}\left(S_{k, n}, Z_{S}\right)+d_{\mathrm{TV}}\left(Z_{S}, Z_{N}\right) \\
& \leqslant c^{\star}\left(n r_{n}^{d}\right)+d_{\mathrm{TV}}\left(Z_{S}, Z_{N}\right) .
\end{aligned}
$$

Finally, Lemma 7.3 implies that

$$
d_{\mathrm{TV}}\left(Z_{S}, Z_{N}\right) \leqslant\left|\mathbb{E}\left\{S_{k, n}\right\}-\mathbb{E}\left\{N_{k, n}\right\}\right|=|\mathbb{E}\{\Delta\}| \leqslant c^{\star}\left(n r_{n}^{d}\right) .
$$

This completes the proof that $d_{\mathrm{TV}}\left(N_{k, n}, Z_{N}\right) \leqslant c^{\star}\left(n r_{n}^{d}\right) \rightarrow 0$. From Theorem 3.1, since $n^{k+1} r_{n}^{d k} \rightarrow \alpha$, we have that $\mathbb{E}\left\{N_{k, n}\right\} \rightarrow \alpha \mu_{k}$. Using the fact that $Z_{N} \sim$ Poisson $\left(\mathbb{E}\left\{N_{k, n}\right\}\right)$, it is easy to see that $d_{\mathrm{TV}}\left(N_{k, n}\right.$, Poisson $\left.\left(\alpha \mu_{k}\right)\right) \rightarrow 0$, which implies convergence in distribution.

Case 3: $n^{k+1} r_{n}^{d k} \rightarrow \infty$

Proof of Theorem 3.2 continued ( $N_{k, n}$ only). We start with the second moment of $N_{k, n}$

$$
\begin{aligned}
\mathbb{E}\left\{N_{k, n}^{2}\right\} & =\mathbb{E}\left\{\sum_{\mathcal{Y}_{1} \subset \mathcal{P}_{n}} \sum_{\mathcal{Y}_{2} \subset \mathcal{P}_{n}} g_{r_{n}}\left(\mathcal{Y}_{1}, \mathcal{P}_{n}\right) g_{r_{n}}\left(\mathcal{Y}_{2}, \mathcal{P}_{n}\right)\right\} \\
& =\sum_{j=0}^{k+1} \mathbb{E}\left\{\sum_{\mathcal{Y}_{1} \subset \mathcal{P}_{n}} \sum_{\mathcal{Y}_{2} \subset \mathcal{P}_{n}} g_{r_{n}}\left(\mathcal{Y}_{1}, \mathcal{P}_{n}\right) g_{r_{n}}\left(\mathcal{Y}_{2}, \mathcal{P}_{n}\right) \mathbb{1}\left\{\left|\mathcal{Y}_{1} \cap \mathcal{Y}_{2}\right|=j\right\}\right\} \\
& :=\sum_{j=0}^{k+1} \mathbb{E}\left\{I_{j}\right\} .
\end{aligned}
$$

As in the proof of the previous cases, we have that

$$
\lim _{n \rightarrow \infty}\left(n^{k+1} r_{n}^{d k}\right)^{-1} \mathbb{E} I_{k+1}=\mu_{k}, \quad \lim _{n \rightarrow \infty}\left(n^{k+1} r_{n}^{d k}\right)^{-1} \mathbb{E} I_{j}=0, \quad 1 \leqslant j \leqslant k .
$$

However, in this case, $I_{0}$ requires a different treatment. Recall that our interest is in the variance $-\operatorname{Var}\left(N_{k, n}\right)$. So we have

$$
\begin{aligned}
\operatorname{Var}\left(N_{k, n}^{2}\right) & =\mathbb{E}\left\{N_{k, n}^{2}\right\}-\left(\mathbb{E}\left\{N_{k, n}\right\}\right)^{2} \\
& =\mathbb{E}\left\{I_{k+1}\right\}+\sum_{j=1}^{k} \mathbb{E}\left\{I_{j}\right\}+\left(\mathbb{E}\left\{\widehat{I}_{0}\right\}-\left(\mathbb{E}\left\{N_{k, n}\right\}\right)^{2}\right) .
\end{aligned}
$$

Thus, to complete the proof, we need to show that

$$
\lim _{n \rightarrow \infty}\left(n^{k+1} r_{n}^{d k}\right)^{-1}\left(\mathbb{E}\left\{\widehat{I}_{0}\right\}-\left(\mathbb{E}\left\{\widehat{N}_{k, n}\right\}\right)^{2}\right)=0 .
$$

Applying Corollary B.2, we have

$$
\mathbb{E}\left\{I_{0}\right\}=\left(\frac{n^{k+1}}{(k+1) !}\right)^{2} \mathbb{E}\left\{g_{r_{n}}\left(\mathcal{Y}_{1}^{\prime}, \mathcal{Y}_{12}^{\prime} \cup \mathcal{P}_{n}\right) g_{r_{n}}\left(\mathcal{Y}_{2}^{\prime}, \mathcal{Y}_{12}^{\prime} \cup \mathcal{P}_{n}\right)\right\}_{\mathcal{Y}_{1}^{\prime} \cap \mathcal{Y}_{2}^{\prime}=\emptyset}
$$


where $\mathcal{Y}_{1}^{\prime}$ and $\mathcal{Y}_{2}^{\prime}$ are sets of i.i.d. points with density $f$, independent of $\mathcal{P}_{n}$, and $\mathcal{Y}_{12}^{\prime}=\mathcal{Y}_{1}^{\prime} \cup \mathcal{Y}_{2}^{\prime}$. Similarly, applying Theorem B.1, we have

$$
\mathbb{E}\left\{N_{k, n}\right\}=\frac{n^{k+1}}{(k+1) !} \mathbb{E}\left\{g_{r_{n}}\left(\mathcal{Y}_{1}^{\prime}, \mathcal{Y}_{1}^{\prime} \cup \mathcal{P}_{n}\right)\right\}
$$

Therefore, we can write

$$
\left(\mathbb{E}\left\{N_{k, n}\right\}\right)^{2}=\left(\frac{n^{k+1}}{(k+1) !}\right)^{2} \mathbb{E}\left\{g_{r_{n}}\left(\mathcal{Y}_{1}^{\prime}, \mathcal{Y}_{1}^{\prime} \cup \mathcal{P}_{n}\right) g_{r_{n}}\left(\mathcal{Y}_{2}^{\prime}, \mathcal{Y}_{2}^{\prime} \cup \mathcal{P}_{n}^{\prime}\right)\right\}
$$

where $\mathcal{P}_{n}^{\prime}$ is an independent copy of $\mathcal{P}_{n}$. Set

$$
\Delta:=g_{r_{n}}\left(\mathcal{Y}_{1}^{\prime}, \mathcal{Y}_{12}^{\prime} \cup \mathcal{P}_{n}\right) g_{r_{n}}\left(\mathcal{Y}_{2}^{\prime}, \mathcal{Y}_{12}^{\prime} \cup \mathcal{P}_{n}\right)-g_{r_{n}}\left(\mathcal{Y}_{1}^{\prime}, \mathcal{Y}_{1}^{\prime} \cup \mathcal{P}_{n}\right) g_{r_{n}}\left(\mathcal{Y}_{2}^{\prime}, \mathcal{Y}_{2}^{\prime} \cup \mathcal{P}_{n}^{\prime}\right) .
$$

Showing that $n^{k+1} r_{n}^{-d k} \mathbb{E}\{\Delta\} \rightarrow 0$ will complete the proof. Set

$$
\Delta_{1}=\Delta \cdot \mathbb{1}\left\{B\left(\mathcal{Y}_{1}^{\prime}\right) \cap B\left(\mathcal{Y}_{2}^{\prime}\right) \neq \emptyset\right\}, \quad \Delta_{2}=\Delta \cdot \mathbb{1}\left\{B\left(\mathcal{Y}_{1}^{\prime}\right) \cap B\left(\mathcal{Y}_{2}^{\prime}\right)=\emptyset\right\} .
$$

If $\Delta_{1} \neq 0$ then all the elements in $\mathcal{Y}_{1}^{\prime}$ and $\mathcal{Y}_{2}^{\prime}$ are bounded by a ball of radius $2 r_{n}$. Therefore, using Lemma 5.1,

$$
\mathbb{E}\left\{\Delta_{1}\right\} \leqslant c^{\star} r_{n}^{d(2 k+1)}
$$

Next, note that

$$
\begin{aligned}
\Delta_{2}=h_{r_{n}}\left(\mathcal{Y}_{1}^{\prime}\right) h_{r_{n}}\left(\mathcal{Y}_{2}^{\prime}\right) \mathbb{1}\left\{B\left(\mathcal{Y}_{1}^{\prime}\right) \cap B\left(\mathcal{Y}_{2}^{\prime}\right)=\emptyset\right\} \\
\times\left(\mathbb{1}\left\{\mathcal{P}_{n} \cap B\left(\mathcal{Y}_{1}^{\prime}\right)=\emptyset\right\} \mathbb{1}\left\{\mathcal{P}_{n} \cap B\left(\mathcal{Y}_{2}^{\prime}\right)=\emptyset\right\}\right. \\
\left.\quad-\mathbb{1}\left\{\mathcal{P}_{n} \cap B\left(\mathcal{Y}_{1}^{\prime}\right)=\emptyset\right\} \mathbb{1}\left\{\mathcal{P}_{n}^{\prime} \cap B\left(\mathcal{Y}_{2}^{\prime}\right)=\emptyset\right\}\right) .
\end{aligned}
$$

If $\Delta_{2} \neq 0$, then $B\left(\mathcal{Y}_{1}^{\prime}\right)$ and $B\left(\mathcal{Y}_{2}^{\prime}\right)$ are disjoint. Therefore, given $\mathcal{Y}_{1}^{\prime}$ and $\mathcal{Y}_{2}^{\prime}$, the set $\mathcal{P}_{n} \cap$ $B\left(\mathcal{Y}_{2}^{\prime}\right)$ is independent of the set $\mathcal{P}_{n} \cap B\left(\mathcal{Y}_{1}^{\prime}\right)$ (by the spatial independence of the Poisson process), and has the same distribution as $\mathcal{P}_{n}^{\prime} \cap B\left(\mathcal{Y}_{2}^{\prime}\right)$. Thus, $\mathbb{E}\left\{\Delta_{2} \mid \mathcal{Y}_{1}^{\prime}, \mathcal{Y}_{2}^{\prime}\right\}=0$, which implies that $\mathbb{E}\left\{\Delta_{2}\right\}=0$.

To conclude, $\mathbb{E}\{\Delta\} \leqslant c^{\star} r_{n}^{d(2 k+1)}$. Therefore,

$$
\lim _{n \rightarrow \infty} n^{k+1} r_{n}^{-d k} \mathbb{E}\{\Delta\} \leqslant \lim _{n \rightarrow \infty} c^{\star}\left(n r_{n}^{d}\right)^{k+1}=0 .
$$

This completes the proof for the limit variance.

Next, we wish to prove the CLT in Theorem 3.3.

Proof of Theorem 3.3-Part 3. The proof is based on the normal approximation for sums of dependent variables given by Stein's method (Appendix C). We start by counting only critical points located in a compact $A \subset \mathbb{R}^{d}$ for which $\int_{A} f(x) d x>0$. For a fixed $n$, let $\left\{Q_{i, n}\right\}_{i \in \mathbb{N}}$ be a partition of $\mathbb{R}^{d}$ into cubes of side $r_{n}$, and let $I_{A} \subset \mathbb{N}$ be the (finite) set of indices $i$ for which $Q_{i, n} \cap A \neq \emptyset$. For $i \in I_{A}$, set

$$
g_{r_{n}}^{(i)}\left(\mathcal{Y}, \mathcal{P}_{n}\right):=g_{r_{n}}\left(\mathcal{Y}, \mathcal{P}_{n}\right) \mathbb{1}_{A \cap Q_{i, n}}(C(\mathcal{Y})),
$$

where $C(\mathcal{Y})$ is the critical point in $\mathbb{R}^{d}$ generated by $\mathcal{Y}$ (cf. (2.2)). That is, $g_{r_{n}}^{(i)}=1$ 
implies that $\mathcal{Y}$ generates a critical point located in $A \cap Q_{i, n}$. Then

$$
N_{k, n}^{(i)}:=\sum_{\mathcal{Y} \subset \mathcal{P}_{n}} g_{r_{n}}^{(i)}\left(\mathcal{Y}, \mathcal{P}_{n}\right)
$$

is the number of critical points inside $A \cap Q_{i, n}$, and

$$
N_{k, n}^{A}:=\#\left\{\text { critical points of } d_{\mathcal{P}_{n}} \text { inside } A\right\}=\sum_{i \in I_{A}} N_{k, n}^{(i)} .
$$

First, as in the proof of Theorem 3.2, one can show that

$$
\mu_{k}(A):=\lim _{n \rightarrow \infty}\left(n^{k+1} r_{n}^{d k}\right)^{-1} \operatorname{Var}\left(N_{k, n}^{A}\right) \in(0, \infty) .
$$

Now, for $i, j \in I_{A}$, define the relation $i \sim j$ if the distance between $Q_{i, n}$ and $Q_{j, n}$ is less than $2 r_{n}$. Then $\left(I_{A}, \sim\right)$ is the dependency graph (cf. (C.1)) for the set $\left\{N_{k, n}^{(i)}\right\}_{i \in I_{A}}$. This follows from the fact that a critical point located inside $Q_{i, n}$ is generated by points of $\mathcal{P}_{n}$ that are within distance $r_{n}$ from $Q_{i, n}$ (along with the spatial independence of $\mathcal{P}_{n}$ ). The degree of this graph is bounded by $5^{d}$. Consider the normalized random variables

$$
\xi_{i}:=\frac{N_{k, n}^{(i)}-\mathbb{E}\left\{N_{k, n}^{(i)}\right\}}{\left(\operatorname{Var}\left(N_{k, n}^{A}\right)\right)^{1 / 2}} .
$$

According to Theorem C.3, in order to prove a CLT for $N_{k, n}^{A}$, all we have to do now is to find bounds for $\mathbb{E}\left\{\left|\xi_{i}\right|^{p}\right\}, p=3,4$.

Let $B_{r_{n}}\left(Q_{i, n}\right) \subset \mathbb{R}^{d}$ be the set of points within distance $r_{n}$ of $Q_{i, n}$, and let $Z_{i}:=$ $\left|\mathcal{P}_{n} \cap B_{r_{n}}\left(Q_{i, n}\right)\right|$ be the number of points of the Poisson process $\mathcal{P}_{n}$ lying inside $B_{r_{n}}\left(Q_{i, n}\right)$. Then $Z_{i} \sim \operatorname{Poisson}\left(\lambda_{i}\right)$ where $\lambda_{i}=\int_{B_{r_{n}}\left(Q_{i, n}\right)} n f(x) d x \leqslant n f_{\max }\left(3 r_{n}\right)^{d}$. Thus, $Z_{i}$ is stochastically dominated by a Poisson random variable with parameter $c^{\star} n r_{n}^{d}$. Now,

$$
N_{k, n}^{(i)} \leqslant\left(\begin{array}{c}
Z_{i} \\
k+1
\end{array}\right) \leqslant c^{\star} Z_{i}^{k+1}
$$

Therefore, for any $p \geqslant 1$,

$$
\mathbb{E}\left\{\left|N_{k, n}^{(i)}\right|^{p}\right\} \leqslant c^{\star} \mathbb{E}\left\{Z_{i}^{p(k+1)}\right\} \leqslant c^{\star}\left(n r_{n}^{d}\right)^{p(k+1)} \leqslant c^{\star}\left(n r_{n}^{d}\right)^{k+1},
$$

since $n r_{n}^{d}$ is bounded (note that each of the $c^{\star}$ 's stands for a different value). Thus, it is easy to show that also

$$
\mathbb{E}\left\{\left|N_{k, n}^{(i)}-\mathbb{E}\left\{N_{k, n}^{(i)}\right\}\right|^{p}\right\} \leqslant c^{\star}\left(n r_{n}^{d}\right)^{k+1} .
$$

Since $A$ is compact, there exists a constant $v$ such that $\left|I_{A}\right| \leqslant v r_{n}^{-d}$. Therefore, for $p=3,4$,

$$
\sum_{i \in I_{A}} \mathbb{E}\left\{\left|\xi_{i}\right|^{p}\right\} \leqslant \frac{v r_{n}^{-d} c^{\star}\left(n r_{n}^{d}\right)^{k+1}}{\left(\operatorname{Var}\left(N_{k, n}^{A}\right)\right)^{p / 2}}=v c^{\star}\left(n^{k+1} r_{n}^{d k}\right)^{1-p / 2}\left(\frac{\left(n^{k+1} r_{n}^{d k}\right)}{\operatorname{Var}\left(N_{k, n}^{A}\right)}\right)^{p / 2} \rightarrow 0,
$$

where we used the fact that $n^{k+1} r_{n}^{d k} \rightarrow \infty$, and the limit in Theorem 3.2. From 
Theorem C.3, we conclude that

$$
\frac{N_{k, n}^{A}-\mathbb{E}\left\{N_{k, n}^{A}\right\}}{\left(\operatorname{Var}\left(N_{k, n}^{A}\right)\right)^{1 / 2}} \stackrel{\mathcal{L}}{\rightarrow} \mathcal{N}(0,1) .
$$

Now that we have a CLT for $N_{k, n}^{A}$, we need to extend it to one for $\widehat{N}_{k, n}$. The method we shall use is exactly the same as the one used in [23], but, for completeness, we nevertheless include it.

Set $A_{M}=[-M, M]^{d}, A^{M}=\mathbb{R}^{d} \backslash A_{M}$, and suppose that $M$ is large enough such that $\int_{A_{M}} f(z) d z>0$. Set

$$
\zeta_{n}(A)=\frac{N_{k, n}^{A}-\mathbb{E}\left\{N_{k, n}^{A}\right\}}{\left(n^{k+1} r_{n}^{d k}\right)^{1 / 2}} \quad \zeta_{n}=\frac{N_{k, n}-\mathbb{E}\left\{N_{k, n}\right\}}{\left(n^{k+1} r_{n}^{d k}\right)^{1 / 2}} .
$$

To complete the proof we need to show that $\left|\mathbb{P}\left(\zeta_{n} \leqslant t\right)-\Phi\left(t / \sqrt{\mu_{k}}\right)\right| \rightarrow 0$, where $\Phi(\cdot)$ is the standard normal distribution function. Clearly, $\zeta_{n}=\zeta_{n}\left(A_{M}\right)+\zeta_{n}\left(A^{M}\right)$, and from (7.9) we have that

$$
\zeta_{n}\left(A_{M}\right) \stackrel{\mathcal{L}}{\rightarrow} \mathcal{N}\left(0, \mu_{k}\left(A_{M}\right)\right) .
$$

For every $t \in \mathbb{R}$ and $M, \delta>0$ we have

$$
\begin{aligned}
\left|\mathbb{P}\left(\zeta_{n} \leqslant t\right)-\Phi\left(t / \sqrt{\mu_{k}}\right)\right| \leqslant & \left|\mathbb{P}\left(\zeta_{n} \leqslant t\right)-\mathbb{P}\left(\zeta_{n}\left(A_{M}\right) \leqslant t-\delta\right)\right| \\
& +\left|\mathbb{P}\left(\zeta_{n}\left(A_{M}\right) \leqslant t-\delta\right)-\Phi\left((t-\delta) / \sqrt{\mu_{k}\left(A_{M}\right)}\right)\right| \\
& +\left|\Phi\left((t-\delta) / \sqrt{\mu_{k}\left(A_{M}\right)}\right)-\Phi\left(t / \sqrt{\mu_{k}}\right)\right| .
\end{aligned}
$$

Now,

$$
\begin{aligned}
\mathbb{P}\left(\zeta_{n} \leqslant t\right)=\mathbb{P}\left(\zeta_{n}\left(A_{M}\right) \leqslant t-\delta, \zeta_{n} \leqslant t\right) & +\mathbb{P}\left(\left|\zeta_{n}\left(A_{M}\right)-t\right|<\delta, \zeta_{n} \leqslant t\right) \\
& +\mathbb{P}\left(\zeta_{n}\left(A_{M}\right) \geqslant t+\delta, \zeta_{n} \leqslant t\right) .
\end{aligned}
$$

Note that the first term equals

$$
\mathbb{P}\left(\zeta_{n}\left(A_{M}\right) \leqslant t-\delta\right)-\mathbb{P}\left(\zeta_{n}\left(A_{M}\right) \leqslant t-\delta, \zeta_{n}>t\right) .
$$

Thus,

$$
\begin{aligned}
& \left|\mathbb{P}\left(\zeta_{n} \leqslant t\right)-\mathbb{P}\left(\zeta_{n}\left(A_{M}\right) \leqslant t-\delta\right)\right| \leqslant \mathbb{P}\left(\zeta_{n}\left(A_{M}\right) \leqslant t-\delta, \zeta_{n}>t\right) \\
& \quad+\mathbb{P}\left(\left|\zeta_{n}\left(A_{M}\right)-t\right|<\delta, \zeta_{n} \leqslant t\right)+\mathbb{P}\left(\zeta_{n}\left(A_{M}\right) \geqslant t+\delta, \zeta_{n} \leqslant t\right) \\
& \quad \leqslant \mathbb{P}\left(\left|\zeta_{n}\left(A^{M}\right)\right|>\delta\right)+\mathbb{P}\left(\left|\zeta_{n}\left(A_{M}\right)-t\right|<\delta\right) .
\end{aligned}
$$

From Chebyshev's inequality we have that $\mathbb{P}\left(\left|\zeta_{n}\left(A^{M}\right)\right|>\delta\right) \leqslant \delta^{-2} \operatorname{Var}\left(\zeta_{n}\left(A^{M}\right)\right)$. From (7.10), we have that

$$
\begin{aligned}
\lim _{n \rightarrow \infty} \mathbb{P}\left(\left|\zeta_{n}\left(A_{M}\right)-t\right|<\delta\right) & =\Phi\left((t+\delta) / \sqrt{\mu_{k}\left(A_{M}\right)}\right)-\Phi\left((t-\delta) / \sqrt{\mu_{k}\left(A_{M}\right)}\right) \\
& \leqslant \frac{2 \delta}{\sqrt{2 \pi \mu_{k}\left(A_{M}\right)}} .
\end{aligned}
$$


Therefore,

$$
\limsup _{n \rightarrow \infty}\left|\mathbb{P}\left(\zeta_{n} \leqslant t\right)-\mathbb{P}\left(\zeta_{n}\left(A_{M}\right) \leqslant t-\delta\right)\right| \leqslant \frac{\mu_{k}\left(A^{M}\right)}{\delta^{2}}+\frac{2 \delta}{\sqrt{2 \pi \mu_{k}\left(A_{M}\right)}} .
$$

For $\epsilon>0$, choose $\delta=\epsilon \sqrt{\pi \mu_{k}} / 4$. Since $\lim _{M \rightarrow \infty} \mu_{k}\left(A_{M}\right)=\mu_{k}$, and $\lim _{M \rightarrow \infty} \mu_{k}\left(A^{M}\right)$ $=0$, there exists $M$ large enough such that $\mu_{k}\left(A_{M}\right) \geqslant \mu_{k} / 2, \mu_{k}\left(A^{M}\right) \leqslant \epsilon \delta^{2} / 2$, and also $\left|\Phi\left((t-\delta) / \sqrt{\mu_{k}\left(A_{M}\right)}\right)-\Phi\left(t / \sqrt{\mu_{k}}\right)\right|<2 \epsilon$. For this choice of $\delta, M$, using last displayed inequality, we have

$$
\limsup _{n \rightarrow \infty}\left|\mathbb{P}\left(\zeta_{n} \leqslant t\right)-\mathbb{P}\left(\zeta_{n}\left(A_{M}\right) \leqslant t-\delta\right)\right| \leqslant \epsilon
$$

Finally, returning to (7.11), there exists $N>0$ such that for every $n>N$,

$$
\left|\mathbb{P}\left(\zeta_{n} \leqslant t\right)-\Phi\left(t / \sqrt{\mu_{k}}\right)\right|<4 \epsilon .
$$

This completes the proof.

\section{The critical and supercritical ranges $\left(n r_{n}^{d} \rightarrow \lambda \in(0, \infty]\right)$}

We start with the expectation computations. The following standard lemma is going to play a key role in the supercritical regime.

Lemma 8.1. Let $D \subset \mathbb{R}^{d}$ be a compact convex set with positive Lebesgue measure, and let $B_{r}(x) \subset \mathbb{R}^{d}$ be the ball of radius $r$ around $x$. Then there exists a constant $c^{\star}$ such that for every $r<\operatorname{diam}(D)$ and $x \in D$,

$$
\operatorname{Vol}\left(B_{r}(x) \cap D\right) \geqslant c^{\star} r^{d} .
$$

The following lemma is analogous to Lemma 6.1.

Lemma 8.2. Let $\mathcal{Y}$ be a set of $k+1$ i.i.d. random variables with density $f$, independent of the Poisson process $\mathcal{P}_{n}$. Then,

$$
\lim _{n \rightarrow \infty} n^{k} \mathbb{E}\left\{g_{r_{n}}\left(\mathcal{Y}, \mathcal{Y} \cup \mathcal{P}_{n}\right)\right\}=(k+1) ! \gamma_{k}(\lambda) .
$$

Proof. Setting $s_{n}=n^{-1 / d}$ and mimicking the proof of Lemma 6.1, we obtain

$$
\begin{aligned}
\mathbb{E} & \left\{g_{r_{n}}\left(\mathcal{Y}, \mathcal{Y} \cup \mathcal{P}_{n}\right)\right\}=\int_{\left(\mathbb{R}^{d}\right)^{k+1}} f(\mathbf{x}) h_{r_{n}}(\mathbf{x}) e^{-n p(\mathbf{x})} d \mathbf{x} \\
& =s_{n}^{d k} \int_{\mathbb{R}^{d}} \int_{\left(\mathbb{R}^{d}\right)^{k}} f(x) f\left(x+s_{n} \mathbf{y}\right) h_{r_{n}}\left(x, x+s_{n} \mathbf{y}\right) e^{-n p\left(x, x+s_{n} \mathbf{y}\right)} d \mathbf{y} d x \\
& =n^{-k} \int_{\mathbb{R}^{d}} f(x) \int_{\left(\mathbb{R}^{d}\right)^{k}} f\left(x+s_{n} \mathbf{y}\right) h_{\tau_{n}}(0, \mathbf{y}) e^{-n p\left(x, x+s_{n} \mathbf{y}\right)} d \mathbf{y} d x,
\end{aligned}
$$

where $\tau_{n}=r_{n} / s_{n}=n^{1 / d} r_{n}$. We wish to apply the dominated convergence theorem for the last integral. Thus, we need to bound the integrand with an integrable expression.

In the critical range this is done much as in the subcritical range. Since $n r_{n}^{d} \rightarrow \lambda<$ $\infty$, we have that $\tau_{n}$ is bounded by some value $M$. Now, for $h_{\tau_{n}}(0, \mathbf{y})$ to be nonzero, 
all the elements $y_{1}, \ldots, y_{k} \in \mathbb{R}^{d}$ must lie inside $B_{2 \tau_{n}}(0) \subset B_{2 M}(0)$. Therefore,

$$
\left|f\left(x+s_{n} \mathbf{y}\right) h_{\tau_{n}}(0, \mathbf{y}) e^{-n p\left(x, x+s_{n} \mathbf{y}\right)}\right| \leqslant f_{\max }^{k} \mathbb{1}_{B_{2 M}(0)}\left(y_{1}\right) \cdots \mathbb{1}_{B_{2 M}(0)}\left(y_{k}\right),
$$

and this expression is integrable.

The last argument cannot be applied in the supercritical range, since then $\tau_{n}$ is no longer bounded. However, applying our additional lower boundedness assumptions on the $f$, we can proceed as follows. Since we now have $f_{\min }>0$, we also have that

$$
p(\mathbf{x})=\int_{B(\mathbf{x})} f(z) d z \geqslant f_{\min } \operatorname{Vol}(B(\mathbf{x}) \cap \operatorname{supp}(f)) .
$$

If $h_{r_{n}}(\mathbf{x}) \neq 0$, then necessarily $C(\mathbf{x}) \in \operatorname{conv}^{\circ}(\mathbf{x})$ and $R(\mathbf{x}) \leqslant r_{n}$ (cf. (2.6)). In addition, if $f(\mathbf{x}) \neq 0$, then $\mathbf{x} \subset \operatorname{supp}(f)$. Since we assume that $\operatorname{supp}(f)$ is convex, we have that $C(\mathbf{x}) \in \operatorname{supp}(f)$ as well. Thus, $B(\mathbf{x})$ is a ball centered at $C(\mathbf{x}) \in \operatorname{supp}(f)$, with radius $R(\mathbf{x})$ small enough, and Lemma 8.1 yields

$$
\operatorname{Vol}(B(\mathbf{x}) \cap \operatorname{supp}(f)) \geqslant c^{\star} R^{d}(\mathbf{x}) .
$$

Using the inequality in (8.2), and the definition of $R(\mathbf{x})$ in (2.3), we have that

$$
p\left(x, x+s_{n} \mathbf{y}\right) \geqslant f_{\min } c^{\star} R\left(x, x+s_{n} \mathbf{y}\right)=f_{\min } c^{\star} s_{n}^{d} R^{d}(0, \mathbf{y})=f_{\min } c^{\star} n^{-1} R^{d}(0, \mathbf{y}) .
$$

This can be used to bound the integrand in (8.1), so that

$$
\begin{aligned}
\left|f\left(x+s_{n} \mathbf{y}\right) h_{\tau_{n}}(0, \mathbf{y}) e^{-n p\left(x, x+s_{n} \mathbf{y}\right)}\right| & \leqslant f_{\max }^{k} e^{-n f_{\min } c^{\star} R^{d}\left(x, x+s_{n} \mathbf{y}\right)} \\
& =f_{\max }^{k} e^{-f_{\min c^{\star} R^{d}(0, \mathbf{y})}}
\end{aligned}
$$

Next, note that for $i=1, \ldots, k, R(0, \mathbf{y}) \geqslant\left\|y_{i}\right\| / 2$. Thus,

$$
R^{d}(0, \mathbf{y}) \geqslant \frac{1}{2^{d} k} \sum_{j=1}^{k}\left\|y_{j}\right\|^{d},
$$

which implies that the expression in (8.3) is indeed integrable, and so the DCT can be safely applied in both regimes.

Next, we compute the limit of the integral in (8.1). Note first that

$$
\begin{aligned}
n p\left(x, x+s_{n} \mathbf{y}\right) & =n \int_{B\left(x, x+s_{n} \mathbf{y}\right)} f(z) d z \\
& =n \operatorname{Vol}\left(B\left(x, x+s_{n} \mathbf{y}\right)\right) \frac{\int_{B\left(x, x+s_{n} \mathbf{y}\right)} f(z) d z}{\operatorname{Vol}\left(B\left(x, x+s_{n} \mathbf{y}\right)\right)} \\
& =n \omega_{d}\left(s_{n} R(0, \mathbf{y})\right)^{d} \frac{\int_{B\left(x, x+s_{n} \mathbf{y}\right)} f(z) d z}{\operatorname{Vol}\left(B\left(x, x+s_{n} \mathbf{y}\right)\right)} \\
& =\omega_{d} R^{d}(0, \mathbf{y}) \frac{\int_{B\left(x, x+s_{n} \mathbf{y}\right)} f(z) d z}{\operatorname{Vol}\left(B\left(x, x+s_{n} \mathbf{y}\right)\right)},
\end{aligned}
$$

and using the Lebesgue differentiation theorem yields

$$
\lim _{n \rightarrow \infty} n p\left(x, x+s_{n} \mathbf{y}\right)=\omega_{d} R^{d}(0, \mathbf{y}) f(x) .
$$


Taking the limit of all the other terms in (8.1), we have

$$
\lim _{n \rightarrow \infty} n^{k} \mathbb{E}\left\{g_{r_{n}}\left(\mathcal{Y}, \mathcal{Y} \cup \mathcal{P}_{n}\right)\right\}=\int_{\left(\mathbb{R}^{d}\right)^{k+1}} f^{k+1}(x) h_{\tau_{\infty}}(0, \mathbf{y}) e^{-\omega_{d} R^{d}(0, \mathbf{y}) f(x)} d \mathbf{y} d x,
$$

where $\tau_{\infty}=\lim _{n \rightarrow \infty} \tau_{n}$. In the supercritical regime, $\tau_{\infty}=\infty$, and consequently $h_{\tau_{\infty}}(\cdot)=h_{\infty}(\cdot)=h(\cdot)$. Thus,

$$
\begin{aligned}
\lim _{n \rightarrow \infty} n^{k} \mathbb{E}\left\{g_{r_{n}}\left(\mathcal{Y}, \mathcal{Y} \cup \mathcal{P}_{n}\right)\right\} & =\int_{\left.\mathbb{R}^{d}\right)^{k+1}} f^{k+1}(x) h(0, \mathbf{y}) e^{-\omega_{d} R^{d}(0, \mathbf{y}) f(x)} d \mathbf{y} d x \\
& =(k+1) ! \gamma_{k}(\infty)
\end{aligned}
$$

In the critical range, $\tau_{n} \rightarrow \lambda^{1 / d}$. Therefore,

$$
\begin{aligned}
& \lim _{n \rightarrow \infty} n^{k} \mathbb{E}\left\{g_{r_{n}}\left(\mathcal{Y}, \mathcal{Y} \cup \mathcal{P}_{n}\right)\right\}=\int_{\left(\mathbb{R}^{d}\right)^{k+1}} f^{k+1}(x) h_{\lambda^{1 / d}}(0, \mathbf{y}) e^{-\omega_{d} R^{d}(0, \mathbf{y}) f(x)} d \mathbf{y} d x \\
& \quad=\lambda^{k} \int_{\left(\mathbb{R}^{d}\right)^{k+1}} f^{k+1}(x) h_{\lambda^{1 / d}}\left(0, \lambda^{1 / d} \mathbf{z}\right) e^{-\lambda \omega_{d} R^{d}(0, \mathbf{z}) f(x)} d \mathbf{z} d x=(k+1) ! \gamma_{k}(\lambda) .
\end{aligned}
$$

This completes the proof.

\subsection{Asymptotic means}

Using Lemma 8.2, we can prove Theorem 3.5.

Proof of Theorem 3.5. Using Theorem B.1,

$$
\mathbb{E}\left\{N_{k, n}\right\}=\frac{n^{k+1}}{(k+1) !} \mathbb{E}\left\{g_{r_{n}}\left(\mathcal{Y}^{\prime}, \mathcal{Y}^{\prime} \cup \mathcal{P}_{n}\right)\right\}
$$

and, using Lemma 8.2,

$$
\lim _{n \rightarrow \infty} n^{-1} \mathbb{E}\left\{N_{k, n}\right\}=\gamma_{k}(\lambda)
$$

which completes the proof.

\subsection{Asymptotic variance}

Proof of Theorem 3.6. As in the proof of Theorem 3.2,

$$
\operatorname{Var}\left(N_{k, n}^{2}\right)=\mathbb{E}\left\{N_{k, n}\right\}+\sum_{j=1}^{k} \mathbb{E}\left\{I_{j}\right\}+\left(\mathbb{E}\left\{I_{0}\right\}-\left(\mathbb{E}\left\{N_{k, n}\right\}\right)^{2}\right),
$$

where

$$
I_{j}=\sum_{\mathcal{Y}_{1} \subset \mathcal{P}_{n}} \sum_{\mathcal{Y}_{2} \subset \mathcal{P}_{n}} g_{r_{n}}\left(\mathcal{Y}_{1}, \mathcal{P}_{n}\right) g_{r_{n}}\left(\mathcal{Y}_{2}, \mathcal{P}_{n}\right) \mathbb{1}\left\{\left|\mathcal{Y}_{1} \cap \mathcal{Y}_{2}\right|=j\right\}
$$

From Corollary B.2,

$$
\mathbb{E}\left\{I_{j}\right\}=\frac{n^{2 k+2-j}}{j !((k+1-j) !)^{2}} \mathbb{E}\left\{g_{r_{n}}\left(\mathcal{Y}_{1}^{\prime}, \mathcal{Y}_{12}^{\prime} \cup \mathcal{P}_{n}\right) g_{r_{n}}\left(\mathcal{Y}_{2}^{\prime}, \mathcal{Y}_{12}^{\prime} \cup \mathcal{P}_{n}\right)\right\}_{\left|\mathcal{Y}_{1}^{\prime} \cap \mathcal{Y}_{2}^{\prime}\right|=j}
$$


For $0<j<k+1$, as in the proof of Lemma 8.2, one can show that

$$
\begin{gathered}
\lim _{n \rightarrow \infty} n^{d(2 k+1-j)} \mathbb{E}\left\{g_{r_{n}}\left(\mathcal{Y}_{1}^{\prime}, \mathcal{Y}_{12}^{\prime} \cup \mathcal{P}_{n}\right) g_{r_{n}}\left(\mathcal{Y}_{2}^{\prime}, \mathcal{Y}_{12}^{\prime} \cup \mathcal{P}_{n}\right)\right\}\left|\mathcal{Y}_{1}^{\prime} \cap \mathcal{Y}_{2}^{\prime}\right|=j \\
=\int_{\mathbb{R}^{d(2 k+2-j)}} f^{2 k+2-j}(x) h_{\tau_{\infty}}\left(0, \mathbf{y}_{1} \cup \mathbf{z}\right) h_{\tau_{\infty}}\left(0, \mathbf{y}_{2} \cup \mathbf{z}\right) \\
\times e^{-\operatorname{Vol}\left(B\left(0, \mathbf{y}_{1} \cup \mathbf{z}\right) \cup B\left(0, \mathbf{y}_{2} \cup \mathbf{z}\right)\right) f(x)} d x d \mathbf{y}_{1} d \mathbf{y}_{2} d \mathbf{z}
\end{gathered}
$$

where $x \in \mathbb{R}^{d}, \mathbf{y}_{i} \in \mathbb{R}^{d(k+1-j)}, \mathbf{z} \in \mathbb{R}^{d(j-1)}$, and $\tau_{\infty}=\lim _{n \rightarrow \infty} n^{1 / d} r_{n}$. Therefore,

$$
\lim _{n \rightarrow \infty} n^{-1} \mathbb{E}\left\{I_{j}\right\}=\gamma_{k}^{(j)}(\lambda)
$$

where

$$
\begin{aligned}
\gamma_{k}^{(j)}(\lambda):=\frac{\lambda^{2 k+1-j}}{j !((k+1-j) !)^{2}} & \int_{\mathbb{R}^{d(2 k+2-j)}} f^{2 k+2-j}(x) h_{1}\left(0, \mathbf{y}_{1} \cup \mathbf{z}\right) h_{1}\left(0, \mathbf{y}_{2} \cup \mathbf{z}\right) \\
& \times e^{-\lambda \operatorname{Vol}\left(B\left(0, \mathbf{y}_{1} \cup \mathbf{z}\right) \cup B\left(0, \mathbf{y}_{2} \cup \mathbf{z}\right)\right) f(x)} d x d \mathbf{y}_{1} d \mathbf{y}_{2} d \mathbf{z}
\end{aligned}
$$

for $\lambda \in(0, \infty)$, and

$$
\begin{aligned}
\gamma_{k}^{(j)}(\infty):=\frac{1}{j !((k+1-j) !)^{2}} & \int_{\mathbb{R}^{d(2 k+2-j)}} f^{2 k+2-j}(x) h\left(0, \mathbf{y}_{1} \cup \mathbf{z}\right) h\left(0, \mathbf{y}_{2} \cup \mathbf{z}\right) \\
& \times e^{-\operatorname{Vol}\left(B\left(0, \mathbf{y}_{1} \cup \mathbf{z}\right) \cup B\left(0, \mathbf{y}_{2} \cup \mathbf{z}\right)\right) f(x)} d x d \mathbf{y}_{1} d \mathbf{y}_{2} d \mathbf{z} .
\end{aligned}
$$

It is easy to show that $0<\gamma_{k}^{j}(\lambda)<\infty$ for $\lambda \in(0, \infty]$. For $j=0$, we define

$$
\Delta:=g_{r_{n}}\left(\mathcal{Y}_{1}^{\prime}, \mathcal{Y}_{12}^{\prime} \cup \mathcal{P}_{n}\right) g_{r_{n}}\left(\mathcal{Y}_{2}^{\prime}, \mathcal{Y}_{12}^{\prime} \cup \mathcal{P}_{n}\right)-g_{r_{n}}\left(\mathcal{Y}_{1}^{\prime}, \mathcal{Y}_{1}^{\prime} \cup \mathcal{P}_{n}\right) g_{r_{n}}\left(\mathcal{Y}_{2}^{\prime}, \mathcal{Y}_{2}^{\prime} \cup \mathcal{P}_{n}^{\prime}\right)
$$

so that

$$
\mathbb{E}\left\{I_{0}\right\}-\left(\mathbb{E}\left\{N_{k, n}\right\}\right)^{2}=\frac{n^{2 k+2}}{((k+1) !)^{2}} \mathbb{E}\{\Delta\}
$$

Now set

$$
\Delta_{1}=\Delta \cdot \mathbb{1}\left\{B\left(\mathcal{Y}_{1}^{\prime}\right) \cap B\left(\mathcal{Y}_{2}^{\prime}\right) \neq \emptyset\right\}, \quad \Delta_{2}=\Delta \cdot \mathbb{1}\left\{B\left(\mathcal{Y}_{1}^{\prime}\right) \cap B\left(\mathcal{Y}_{2}^{\prime}\right)=\emptyset\right\} .
$$

Then, as in the proof of Theorem 3.2, we can show that $\mathbb{E}\left\{\Delta_{2}\right\}=0$, and

$$
\begin{aligned}
& \lim _{n \rightarrow \infty} n^{2 k+1} \mathbb{E}\left\{\Delta_{1}\right\} \\
& =\int_{\mathbb{R}^{d(2 k+2)}} f^{2 k+2}(x) h_{\tau_{\infty}}\left(0, \mathbf{y}_{1}\right) h_{\tau_{\infty}}\left(0, \mathbf{y}_{2}\right) \mathbb{1}\left\{B\left(0, \mathbf{y}_{1}\right) \cap B\left(z, z+\mathbf{y}_{2}\right) \neq \emptyset\right\} \\
& \times\left(e^{-\operatorname{Vol}\left(B\left(0, \mathbf{y}_{1}\right) \cup B\left(z, z+\mathbf{y}_{2}\right)\right) f(x)}-e^{-\omega_{d}\left(R^{d}\left(0, \mathbf{y}_{1}\right)+R^{d}\left(0, \mathbf{y}_{2}\right)\right) f(x)}\right) d x d z d \mathbf{y}_{1} d \mathbf{y}_{2},
\end{aligned}
$$

where $x, z \in \mathbb{R}^{d}$, and $\mathbf{y}_{i} \in\left(\mathbb{R}^{d}\right)^{k}$. Thus,

$$
\lim _{n \rightarrow \infty} n^{-1}\left(\mathbb{E}\left\{I_{0}\right\}-\left(\mathbb{E}\left\{N_{k, n}\right\}\right)^{2}\right)=\gamma_{k}^{(0)}(\lambda),
$$


where

$$
\begin{aligned}
\gamma_{k}^{(0)}(\lambda) & :=\frac{\lambda^{2 k+1}}{((k+1) !)^{2}} \\
& \times \int_{\mathbb{R}^{d(2 k+2)}} f^{2 k+2}(x) h_{1}\left(0, \mathbf{y}_{1}\right) h_{1}\left(0, \mathbf{y}_{2}\right) \mathbb{1}\left\{B\left(0, \mathbf{y}_{1}\right) \cap B\left(z, z+\mathbf{y}_{2}\right) \neq \emptyset\right\} \\
& \times\left(e^{-\lambda \operatorname{Vol}\left(B\left(0, \mathbf{y}_{1}\right) \cup B\left(z, z+\mathbf{y}_{2}\right)\right) f(x)}-e^{-\lambda \omega_{d}\left(R^{d}\left(0, \mathbf{y}_{1}\right)+R^{d}\left(0, \mathbf{y}_{2}\right)\right) f(x)}\right) d x d z d \mathbf{y}_{1} d \mathbf{y}_{2},
\end{aligned}
$$

for $\lambda<\infty$, and

$$
\begin{aligned}
\gamma_{k}^{(0)}(\infty) & :=\frac{1}{((k+1) !)^{2}} \\
& \times \int_{\mathbb{R}^{d(2 k+2)}} f^{2 k+2}(x) h\left(0, \mathbf{y}_{1}\right) h\left(0, \mathbf{y}_{2}\right) \mathbb{1}\left\{B\left(0, \mathbf{y}_{1}\right) \cap B\left(z, z+\mathbf{y}_{2}\right) \neq \emptyset\right\} \\
& \times\left(e^{-\operatorname{Vol}\left(B\left(0, \mathbf{y}_{1}\right) \cup B\left(z, z+\mathbf{y}_{2}\right)\right) f(x)}-e^{-\omega_{d}\left(R^{d}\left(0, \mathbf{y}_{1}\right)+R^{d}\left(0, \mathbf{y}_{2}\right)\right) f(x)}\right) d x d z d \mathbf{y}_{1} d \mathbf{y}_{2} .
\end{aligned}
$$

To conclude, we have proven that

$$
\lim _{n \rightarrow \infty} n^{-1} \operatorname{Var}\left(N_{k, n}\right)=\gamma_{k}(\lambda)+\sum_{j=0}^{k} \gamma_{k}^{(j)}(\lambda):=\sigma_{k}^{2}(\lambda) \in(0, \infty),
$$

as required.

\subsection{CLT}

Next, we prove the CLT result in Theorem 3.7, again using Stein's method, as in the proof of Theorem 3.3.

Proof of Theorem 3.7. We start again by counting only critical points located in a compact set $A \subset \mathbb{R}^{d}$, with $\int_{A} f(x) d x>0$.

We define $Q_{i, n}, N_{k, n}^{(i)}, N_{k, n}^{A}, g_{r_{n}}^{(i)},\left(I_{A}, \sim\right)$, and $\xi_{i}$ the same way as in the proof of Theorem 3.3. Then, as in the proof of Theorem 3.6, one can show that

$$
\lim _{n \rightarrow \infty} n^{-1} \operatorname{Var}\left(N_{k, n}^{A}\right) \in(0, \infty) \text {. }
$$

According to Theorem C.3, in order to prove a CLT for $N_{k, n}^{A}$, we need to find bounds for $\mathbb{E}\left\{\left|\xi_{i}\right|^{p}\right\}, p=3,4$. We start with $p=3$.

$$
\mathbb{E}\left\{\left(N_{k, n}^{(i)}-\mathbb{E}\left\{N_{k, n}^{(i)}\right\}\right)^{3}\right\}=\sum_{j=0}^{3}\left(\begin{array}{l}
3 \\
j
\end{array}\right)(-1)^{j}\left(\mathbb{E}\left\{N_{k, n}^{(i)}\right\}\right)^{3-j} \mathbb{E}\left\{\left(N_{k, n}^{(i)}\right)^{j}\right\} .
$$

The computation of the bound here is similar in spirit to the ones we used in the proof of Theorem 3.2, but technically more complicated, and we shall not give details. Rather, we shall suffice with a brief description of the main ideas: Every element in the sum can be expressed as the expectation of a triple sum of the form

$$
\mathbb{E}\left\{\sum_{\mathcal{Y}_{1} \subset \mathcal{P}_{n}^{(1)}} \sum_{\mathcal{Y}_{2} \subset \mathcal{P}_{n}^{(2)}} \sum_{\mathcal{Y}_{3} \subset \mathcal{P}_{n}^{(3)}} g_{r_{n}}^{(i)}\left(\mathcal{Y}_{1}, \mathcal{P}_{n}^{(1)}\right) g_{r_{n}}^{(i)}\left(\mathcal{Y}_{2}, \mathcal{P}_{n}^{(2)}\right) g_{r_{n}}^{(i)}\left(\mathcal{Y}_{3}, \mathcal{P}_{n}^{(3)}\right)\right\}
$$

where each of the Poisson processes can either be equal to one of the others or an independent copy, depending on $j$. As for $\mathbb{E}\left\{\Delta_{2}\right\}$ in the proof of Theorem 3.6, we can 
use Palm theory, collect all the terms in which at least one of the balls $B\left(\mathcal{Y}_{i}\right)$ is disjoint from the others, and show that they cancel each other. For each of the remaining terms, we can show that if $\left|\mathcal{Y}_{1} \cup \mathcal{Y}_{2} \cup \mathcal{Y}_{3}\right|=3 k+3-j$, with $0 \leqslant j \leqslant 3 k+3$, then the relevant part of the sum in (8.6) is bounded by $c^{\star} n^{3 k+3-j} s_{n}^{d(3 k+2-j)} r_{n}^{d}=c^{\star} n r_{n}^{d}$. This bound is achieved using integral evaluations similar to the ones used in the proof of Theorem 3.6, along with the fact that all the points are located within a distance of $r_{n}$ from the cube $Q_{i, n}$. Thus, we have

$$
\mathbb{E}\left\{\left(N_{k, n}^{(i)}-\mathbb{E}\left\{N_{k, n}^{(i)}\right\}\right)^{3}\right\} \leqslant c^{\star} n r_{n}^{d}
$$

Recall that $\left|I_{A}\right| \leqslant c^{\star} r_{n}^{-d}$. Therefore,

$$
\sum_{i \in I_{A}} \mathbb{E}\left\{\left|\xi_{i}\right|^{3}\right\} \leqslant \frac{c^{\star} r_{n}^{-d} n r_{n}^{d}}{\left(\operatorname{Var}\left(N_{k, n}^{A}\right)\right)^{3 / 2}}=\frac{c^{\star} n}{n^{3 / 2}\left(n^{-1} \operatorname{Var}\left(N_{k, n}^{A}\right)\right)^{3 / 2}} \rightarrow 0 .
$$

The proof for $p=4$ is similar. Thus, from Theorem C. 3 we have that

$$
\frac{N_{k, n}^{A}-\mathbb{E}\left\{N_{k, n}^{A}\right\}}{\left(\operatorname{Var}\left(N_{k, n}^{A}\right)\right)^{1 / 2}} \stackrel{\mathcal{L}}{\rightarrow} \mathcal{N}(0,1) .
$$

To conclude the proof, we need to show that the CLT for $N_{k, n}^{A}$ implies a CLT for $N_{k, n}$. This is done exactly as for Part 3 of Theorem 3.3.

The only remaining results in Section 3 that still require proofs relate to the global number of critical points.

Proof of Theorem 3.8. This theorem is proved exactly the same way as Theorems 3.5, 3.6 , and 3.7 are proved in the super-critical phase. The only difference is that, throughout, $h(\mathbf{x})$ replaces $h_{\tau_{n}}(\mathbf{x})$. This, however does not affect any of the results, since in the limit $h_{\tau_{n}}(\mathbf{x}) \rightarrow h(\mathbf{x})$.

Proof of Proposition 3.9. The expected difference between the global and local number of critical points is given by

$$
\begin{aligned}
& \mathbb{E}\left\{N_{k, n}^{(g)}-N_{k, n}\right\} \\
& =\frac{n^{k+1}}{(k+1) !} n^{-k} \int_{\left(\mathbb{R}^{d}\right)^{k+1}} f(x) f\left(x+s_{n} \mathbf{y}\right)\left(h(0, \mathbf{y})-h_{\tau_{n}}(0, \mathbf{y})\right) e^{-n p\left(x, x+s_{n} \mathbf{y}\right)} d \mathbf{y} d x \\
& =\frac{1}{(k+1) !} \int_{\left(\mathbb{R}^{d}\right)^{k+1}} f(x) f\left(x+s_{n} \mathbf{y}\right)\left(h(0, \mathbf{y})-h_{\tau_{n}}(0, \mathbf{y})\right) n e^{-n p\left(x, x+s_{n} \mathbf{y}\right)} d \mathbf{y} d x
\end{aligned}
$$

As in the proof of Theorem 3.5 (cf. (8.3)), we can show that the integrand is bounded 
by

$$
f(x) f_{\max }^{k}\left(h(0, \mathbf{y})-h_{\tau_{n}}(0, \mathbf{y})\right) n e^{-f_{\min } c^{\star} R^{d}(0, \mathbf{y})} .
$$

Now note that if the integrand is nonzero, then $h \neq h_{\tau_{n}}$, and so $R(0, \mathbf{y})>\tau_{n}$. Therefore, $R^{d}(0, \mathbf{y})>1 / 2\left(R^{d}(0, \mathbf{y})+n r_{n}^{d}\right)$, and (8.8) can be replaced by

$$
f(x) f_{\max }^{k}\left(h(0, \mathbf{y})-h_{\tau_{n}}(0, \mathbf{y})\right) e^{-f_{\min } c^{\star} R^{d}(0, \mathbf{y}) / 2} n e^{-f_{\min } c^{\star} n r_{n}^{d} / 2} .
$$

Assuming that $n r_{n}^{d} \geqslant D^{\star} \log n$, with $D^{\star}=\left(f_{\min } c^{\star} / 2\right)^{-1}$, then $n e^{-f_{\min } c^{\star} n r_{n}^{d} / 2} \leqslant 1$ and we obtain an integrable bound for the integrand. Thus, we can apply the DCT to (8.7). Finally, note that the bound we found in (8.9) converges to zero (since $h_{\tau_{n}} \rightarrow h$ ), so we are done.

\section{Euler characteristic results}

Finally, we prove Corollary 4.2 .

Proof of Corollary 4.2. First note that $\mathbb{E}\left\{N_{0, n}\right\}=n$. Thus,

$$
\mathbb{E}\left\{\chi_{n}\right\}=n+\sum_{k=1}^{d}(-1)^{k} \mathbb{E}\left\{N_{k, n}\right\}
$$

The first two cases of the theorem are now obvious consequences of Theorems 3.1 and 3.5. For the third case, using Theorem 3.8, we have

$$
\lim _{n \rightarrow \infty} n^{-1} \chi_{n}=\lim _{n \rightarrow \infty} n^{-1} \sum_{k=0}^{d}(-1)^{k} N_{k, n}^{(g)}
$$

However, since $N_{k, n}^{(g)}$ counts all the critical points in $\mathbb{R}^{d}$, Morse theory implies

$$
\sum_{k=0}^{d}(-1)^{k} N_{k, n}^{(g)}=\chi\left(\mathbb{R}^{d}\right)=1,
$$

and we can conclude that $\lim _{n \rightarrow \infty} n^{-1} \chi_{n}=0$.

If, in addition, $r_{n}^{d}$ satisfies the conditions of Proposition 3.9 (i.e., $n r_{n}^{d} \geqslant D^{\star} \log n$ ), then

$$
0=\lim _{n \rightarrow \infty} \sum_{k=0}^{d}(-1)^{k} \mathbb{E}\left\{N_{k, n}^{(g)}-N_{k, n}\right\}=1-\lim _{n \rightarrow \infty} \chi_{n},
$$

which implies that $\chi_{n} \rightarrow 1$.

\section{Appendix A. Convergence of random variables}

Probability theory uses a number of different notions of convergence. Below we define the ones used in this paper. 
Let $X_{1}, X_{2}, \ldots$ be a sequence of real valued random variables, with the cumulative distribution function (cdf) of $X_{n}$ given by

$$
F_{n}(x)=\mathbb{P}\left(X_{n} \leqslant x\right),
$$

and let $X$ be a random variable with cumulative distribution function $F$.

Definition A.1. $X_{n}$ converges in distribution, or in law to $X$ (denoted by $X_{n} \stackrel{\mathcal{L}}{\rightarrow} X$ ) if

$$
\lim _{n \rightarrow \infty} F_{n}(x)=F(x)
$$

for every $x \in \mathbb{R}$ at which $F(x)$ is continuous.

This type of convergence is also sometimes referred to as "weak convergence."

Definition A.2. $X_{n}$ converges in $L^{p}$ to $X$ (denoted by $X_{n} \stackrel{L^{p}}{\longrightarrow} X$ ) if

$$
\mathbb{E}\left\{\left|X_{n}-X\right|^{p}\right\} \rightarrow 0 .
$$

Finally, let $A$ be a Borel subset of $\mathbb{R}$, and define the probability measures $\mu_{n}$ and $\mu$ by

$$
\mu_{n}(A)=\mathbb{P}\left(X_{n} \in A\right), \quad \mu(A)=\mathbb{P}(X \in A) .
$$

Then the total variation distance between $X_{n}$ and $X$, or between $\mu_{n}$ and $\mu$, is defined as

$$
d_{\mathrm{TV}}\left(X_{n}, X\right) \equiv d_{\mathrm{TV}}\left(\mu_{n}, \mu\right):=\sup _{A}\left|\mathbb{P}\left(X_{n} \in A\right)-\mathbb{P}(X \in A)\right|,
$$

where the supremum is taken over all Borel subsets of $\mathbb{R}$. This distance provides us with the following notion of convergence.

Definition A.3. $X_{n}$ converges in the total variation distance $\left(X_{n} \stackrel{\mathrm{TV}}{\longrightarrow} X\right)$ if

$$
\lim _{n \rightarrow \infty} d_{\mathrm{TV}}\left(X_{n}, X\right)=0 .
$$

Note that both $L^{p}$ and total variation convergence are stronger than convergence in distribution. Further, while convergence in total variation and in distribution actually refer only to convergence of (deterministic) measures and/or cdf's, convergence in $L^{p}$ demands that all the random variables involved are defined on a common probability space, and that the convergence is that of the random variables themselves.

\section{Appendix B. Palm theory for Poisson processes}

This appendix contains a collection of definitions and theorems which are used in the proofs of this paper. Most of the results are cited from [23], although they may not necessarily have originated there. However, for notational reasons we refer the reader to $[\mathbf{2 3}]$, while other resources include $[\mathbf{2 5}, \mathbf{4}]$. The following theorem is very useful when computing expectations related to Poisson processes.

Theorem B.1 (Palm theory for Poisson processes, [23] Theorem 1.6). Let $f$ be a probability density on $\mathbb{R}^{d}$, and let $\mathcal{P}_{n}$ be a Poisson process on $\mathbb{R}^{d}$ with intensity $\lambda_{n}=$ $n f$. Let $h(\mathcal{Y}, \mathcal{X})$ be a measurable function defined for all finite subsets $\mathcal{Y} \subset \mathcal{X} \subset \mathbb{R}^{d}$ with $|\mathcal{Y}|=k$. Then 


$$
\mathbb{E}\left\{\sum_{\mathcal{Y} \subset \mathcal{P}_{n}} h\left(\mathcal{Y}, \mathcal{P}_{n}\right)\right\}=\frac{n^{k}}{k !} \mathbb{E}\left\{h\left(\mathcal{Y}^{\prime}, \mathcal{Y}^{\prime} \cup \mathcal{P}_{n}\right)\right\}
$$

where $\mathcal{Y}^{\prime}$ is a set of $k$ i.i.d. points in $\mathbb{R}^{d}$ with density $f$, independent of $\mathcal{P}_{n}$.

We shall also need the following corollary, which treats second moments:

Corollary B.2. With the notation above, assuming $\left|\mathcal{Y}_{1}\right|=\left|\mathcal{Y}_{2}\right|=k$,

$\mathbb{E}\left\{\sum_{\substack{\mathcal{Y}_{1}, \mathcal{Y}_{2} \subset \mathcal{P}_{n} \\\left|\mathcal{Y}_{1} \cap \mathcal{Y}_{2}\right|=j}} h\left(\mathcal{Y}_{1}, \mathcal{P}_{n}\right) h\left(\mathcal{Y}_{2}, \mathcal{P}_{n}\right)\right\}=\frac{n^{2 k-j}}{j !((k-j) !)^{2}} \mathbb{E}\left\{h\left(\mathcal{Y}_{1}^{\prime}, \mathcal{Y}_{12}^{\prime} \cup \mathcal{P}_{n}\right) h\left(\mathcal{Y}_{2}^{\prime}, \mathcal{Y}_{12}^{\prime} \cup \mathcal{P}_{n}\right)\right\}$

where $\mathcal{Y}_{12}^{\prime}=\mathcal{Y}_{1}^{\prime} \cup \mathcal{Y}_{2}^{\prime}$ is a set of $2 k-j$ i.i.d. points in $\mathbb{R}^{d}$ with density $f(x)$, independent of $\mathcal{P}_{n}$, and $\left|\mathcal{Y}_{1}^{\prime} \cap \mathcal{Y}_{2}^{\prime}\right|=j$.

Proof. Given $\left|\mathcal{P}_{n}\right|=m$, the sum on the LHS is finite. Therefore,

$$
\begin{aligned}
& \mathbb{E}\left\{\sum_{\substack{\mathcal{Y}_{1}, \mathcal{Y}_{2} \subset \mathcal{P}_{n} \\
\left|\mathcal{Y}_{1} \cap \mathcal{Y}_{2}\right|=j}} h\left(\mathcal{Y}_{1}, \mathcal{P}_{n}\right) h\left(\mathcal{Y}_{2}, \mathcal{P}_{n}\right)\left|\mathcal{P}_{n}\right|=m\right\} \\
& \quad=\left(\begin{array}{c}
m \\
2 k-j
\end{array}\right)\left(\begin{array}{c}
2 k-j \\
k
\end{array}\right)\left(\begin{array}{c}
k \\
j
\end{array}\right) \mathbb{E}\left\{h\left(\mathcal{Y}_{1}, \mathcal{P}_{n}\right) h\left(\mathcal{Y}_{2}, \mathcal{P}_{n}\right)|| \mathcal{P}_{n} \mid=m\right\}_{\left|\mathcal{Y}_{1} \cap \mathcal{Y}_{2}\right|=j}
\end{aligned}
$$

Choosing now all possible subsets $\mathcal{Y}$ of size $2 k-j$, and splitting each of them into two arbitrary subsets $\mathcal{Y}_{1}, \mathcal{Y}_{2}$ of size $k$ with $\left|\mathcal{Y}_{1} \cap \mathcal{Y}_{2}\right|=j$, yields

$$
\begin{aligned}
\mathbb{E}\left\{\sum_{\substack{\mathcal{Y} \subset \mathcal{P}_{n} \\
|\mathcal{Y}|=2 k-j}} h\left(\mathcal{Y}_{1}, \mathcal{P}_{n}\right) h\left(\mathcal{Y}_{2}, \mathcal{P}_{n}\right)|| \mathcal{P}_{n} \mid=m\right\} \\
=\left(\begin{array}{c}
m \\
2 k-j
\end{array}\right) \mathbb{E}\left\{h\left(\mathcal{Y}_{1}, \mathcal{P}_{n}\right) h\left(\mathcal{Y}_{2}, \mathcal{P}_{n}\right)|| \mathcal{P}_{n} \mid=m\right\}_{\left|\mathcal{Y}_{1} \cap \mathcal{Y}_{2}\right|=j}
\end{aligned}
$$

Combining (B.1), (B.2), and Theorem B.1 for subsets $\mathcal{Y}$ of size $2 k-j$ yields

$$
\begin{aligned}
\mathbb{E}\left\{\sum_{\substack{\mathcal{Y}_{1}, \mathcal{Y}_{2} \subset \mathcal{P}_{n} \\
\left|\mathcal{Y}_{1} \cap \mathcal{Y}_{2}\right|=j}} h\left(\mathcal{Y}_{1}, \mathcal{P}_{n}\right) h\left(\mathcal{Y}_{2}, \mathcal{P}_{n}\right)\right\} \\
=\left(\begin{array}{c}
2 k-j \\
k
\end{array}\right)\left(\begin{array}{c}
k \\
j
\end{array}\right) \mathbb{E}\left\{\sum_{\substack{\mathcal{Y} \subset \mathcal{P}_{n} \\
|\mathcal{Y}|=2 k-j}} h\left(\mathcal{Y}_{1}, \mathcal{P}_{n}\right) h\left(\mathcal{Y}_{2}, \mathcal{P}_{n}\right)\right\} \\
=\frac{n^{2 k-j}}{j !((k-j) !)^{2}} \mathbb{E}\left\{h\left(\mathcal{Y}_{1}^{\prime}, \mathcal{Y}_{12}^{\prime} \cup \mathcal{P}_{n}\right) h\left(\mathcal{Y}_{2}^{\prime}, \mathcal{Y}_{12}^{\prime} \cup \mathcal{P}_{n}\right)\right\},
\end{aligned}
$$

where $\mathcal{Y}_{12}^{\prime}=\mathcal{Y}_{1}^{\prime} \cup \mathcal{Y}_{2}^{\prime}$ is a set of $2 k-j$ i.i.d. points in $\mathbb{R}^{d}$ with density $f(x)$, independent of $\mathcal{P}_{n}$, and $\left|\mathcal{Y}_{1}^{\prime} \cap \mathcal{Y}_{2}^{\prime}\right|=j$.

\section{Appendix C. Stein's method}

In this paper we heavily used Stein's method to derive limit theorems for the sums of dependent Bernoulli variables. We need both the Poisson and normal approximations, which are presented below. 
Definition C.1. Let $(I, E)$ be a graph. For $i, j \in I$ we denote $i \sim j$ if $(i, j) \in E$. Let $\left\{\xi_{i}\right\}_{i \in I}$ be a set of random variables. We say that $(I, \sim)$ is a dependency graph for $\left\{\xi_{i}\right\}$ if for every $I_{1} \cap I_{2}=\emptyset$, with no edges between $I_{1}$ and $I_{2}$, the set of variables $\left\{\xi_{i}\right\}_{i \in I_{1}}$ is independent of $\left\{\xi_{i}\right\}_{i \in I_{2}}$. We also define the neighborhood of $i$ as $\mathcal{N}_{i}:=$ $\{i\} \cup\{j \in I: j \sim i\}$.

Theorem C.2 (Stein's Method for Bernoulli Variables, Theorem 2.1 in [23]). Let $\left\{\xi_{i}\right\}_{i \in I}$ be a set of Bernoulli random variables, with dependency graph $(I, \sim)$. Let

$$
p_{i}:=\mathbb{E}\left\{\xi_{i}\right\}, \quad p_{i, j}:=\mathbb{E}\left\{\xi_{i} \xi_{j}\right\}, \quad \lambda:=\sum_{i \in I} p_{i}, \quad W:=\sum_{i \in I} \xi_{i}, \quad Z \sim \operatorname{Poisson}(\lambda) .
$$

Then,

$$
d_{\mathrm{TV}}(W, Z) \leqslant \min \left(3, \lambda^{-1}\right)\left(\sum_{i \in I} \sum_{j \in \mathcal{N}_{i} \backslash\{i\}} p_{i j}+\sum_{i \in I} \sum_{j \in \mathcal{N}_{i}} p_{i} p_{j}\right) .
$$

Theorem C.3 (CLT for sums of weakly dependent variables, Theorem 2.4 in [23]). Let $\left(\xi_{i}\right)_{i \in I}$ be a finite collection of random variables, with $\mathbb{E}\left\{\xi_{i}\right\}=0$. Let $(I, \sim)$ be the dependency graph of $\left(\xi_{i}\right)_{i \in I}$, and assume that its maximal degree is $D-1$. Set $W:=\sum_{i \in I} \xi_{i}$, and suppose that $\mathbb{E}\left\{W^{2}\right\}=1$. Then for all $w \in \mathbb{R}$,

$$
\left|F_{W}(w)-\Phi(w)\right| \leqslant 2(2 \pi)^{-1 / 4} \sqrt{D^{2} \sum_{i \in I} \mathbb{E}\left\{\left|\xi_{i}\right|^{3}\right\}}+6 \sqrt{D^{3} \sum_{i \in I} \mathbb{E}\left\{\left|\xi_{i}\right|^{4}\right\}},
$$

where $F_{W}$ is the distribution function of $W$ and $\Phi$ that of a standard Gaussian.

\section{References}

[1] R.J. Adler, O. Bobrowski, M.S. Borman, E. Subag, and S. Weinberger, Persistent homology for random fields and complexes, In Borrowing Strength: theory powering applications - a Festschrift for Lawrence D. Brown, pages 124-143. Inst. Math. Statist., 2010.

[2] R.J. Adler, O. Bobrowski, and S. Weinberger, Crackle: The homology of noise, Discrete Comput. Geom. in print, arXiv:1301.1466, 2014.

[3] L. Aronshtam, N. Linial, T. Luczak, and R. Meshulam, Collapsibility and vanishing of top homology in random simplicial complexes, Discrete Comput. Geom. 49(2), 317-334, 2013.

[4] R. Arratia, L. Goldstein, and L. Gordon, Two moments suffice for Poisson approximations: The Chen-Stein method, Ann. Probab. 17(1), 9-25, 1989.

[5] E. Babson, C. Hoffman, and M. Kahle, The fundamental group of random 2-complexes, J. Amer. Math. Soc. 24(1), 1-28, 2011.

[6] O. Bobrowski, Algebraic topology of random fields and complexes, PhD thesis, 2012.

[7] O. Bobrowski and M.S. Borman, Euler integration of Gaussian random fields and persistent homology, J. Topol. Anal. 4(1), 49-70, 2012.

[8] O. Bobrowski and S. Mukherjee, The topology of probability distributions on manifolds, Probab. Theory Related Fields pages 1-36, 2014. 
[9] K. Borsuk, On the imbedding of systems of compacta in simplicial complexes, Fund. Math. 35(1), 217-234, 1948.

[10] D.C. Cohen, A. Costa, M. Farber, and T. Kappeler, Topology of random 2complexes, Discrete Comput. Geom. 47(1), 117-149, 2012.

[11] V. Gershkovich and H. Rubinstein, Morse theory for min-type functions, Asian J. Math. 1(4), 696-715, 1997.

[12] R. Ghrist, Barcodes: the persistent topology of data, Bull. Amer. Math. Soc. 45(1), 61-75, 2008.

[13] P. Hall, On the coverage of $k$-dimensional space by $k$-dimensional spheres, Ann. Probab. 13(3), 991-1002, 1985.

[14] A. Hatcher, Algebraic Topology, Cambridge University Press, 2002.

[15] S. Janson, Random coverings in several dimensions, Acta Math. 156(1), 83-118, 1986.

[16] M. Kahle, Random geometric complexes, Discrete Comput. Geom. 45(3), 553573, 2011.

[17] M. Kahle and E. Meckes, Limit theorems for Betti numbers of random simplicial complexes, Homology Homotopy Appl. 15(1), 343-374, 2013.

[18] R. Meshulam and N. Wallach, Homological connectivity of random $k$ dimensional complexes, Random Structures Algorithms 34(3), 408-417, 2009.

[19] J.W. Milnor, Morse theory, Princeton Univ. Press, 1963.

[20] M. Morse and S.S. Cairns, Critical Point Theory in Global Analysis and Differential Topology: An Introduction, Academic Press, 1969.

[21] P. Niyogi, S. Smale, and S. Weinberger, A topological view of unsupervised learning from noisy data, SIAM J. Comput. 40(3), 646-663, 2011.

[22] P. Niyogi, S. Smale, and S. Weinberger, Finding the homology of submanifolds with high confidence from random samples, Discrete Comput. Geom., 39(1-3), 419-441, 2008.

[23] M. Penrose, Random Geometric Graphs, volume 5 of Oxford Studies in Probability, Oxford University Press, Oxford, 2003.

[24] N. Pippenger and K. Schleich, Topological characteristics of random triangulated surfaces, Random Structures Algorithms 28(3), 247-288, 2006.

[25] D. Stoyan, W.S. Kendall, and J. Mecke, Stochastic geometry and its applications, Wiley Series in Probability and Mathematical Statistics: Applied Probability and Statistics. John Wiley \& Sons Ltd., Chichester, 1987.

[26] D. Yogeshwaran and R.J. Adler, On the topology of random complexes built over stationary point processes, Ann. Probab. in print. arXiv:1211.0061, 2012.

Omer Bobrowski omer@math.duke.edu

Department of Mathematics, Duke University, 120 Science Drive, Durham, NC 27708

Robert J. Adler robert@ee.technion.ac.il

Department of Electrical Engineering, Technion-Israel Institute of Technology, Haifa, 32000 , Israel 